
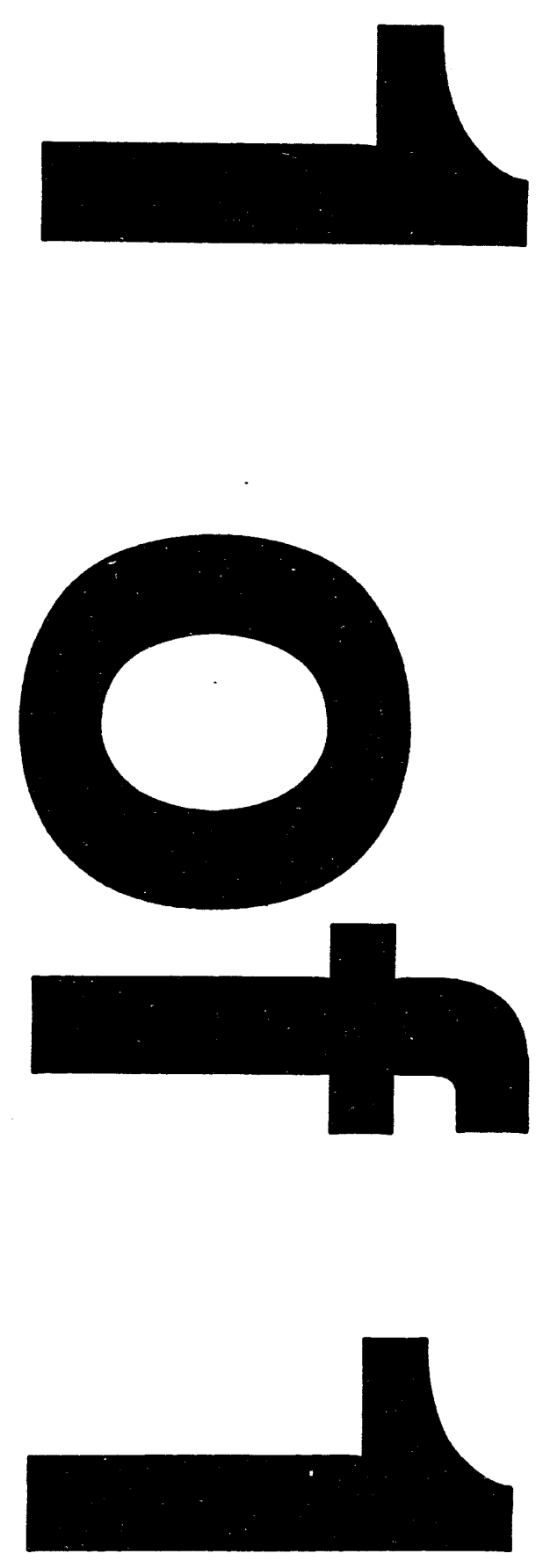


\author{
SYSTEMS OPTIMIZATION LABORATORY \\ DEPARTMENT OF OPERATION RESEARCH \\ STANFORD UNIVERSITY \\ STANFORD, CALIFORNIA 94305-4022
}

\title{
DECOMPOSITION AND (IMPORTANCE) SAMPLING TECHNIQUES FOR MULTI-STAGE STOCHASTIC LINEAR PROGRAMS
}

\author{
b y \\ Gerd Infanger \\ TECHNICAL REPORT SOL 93-7 \\ November 1993

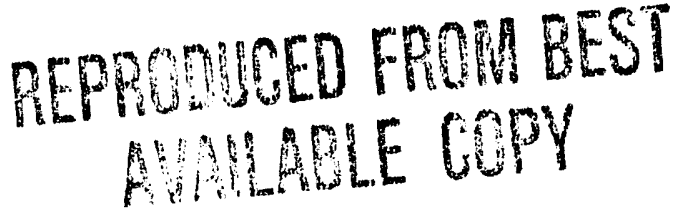

Research leading to this paper was partially supported by the Office of Naval Research Contract N00014-89-J-1659; the National Science Foundation Grants ECS-8906260, DMS-8913089, the Electric Power Research Institute Contract RP 8010-09, CSA-4005335, and the Austrian Science Foundation, "Fonds zur Förderung der wissenschaftlichen Forschung," Grant J0323-Phy.

Any opinions, findings and conclusions or recommendations expressed in this publication are those of the author and do NOT necessarily reflect the views of the above sponsors.

Reproduction in whole or in part is permitted for any purposes of the United States Government. This document has been approved for public release and sale; its distribution is unlimited.

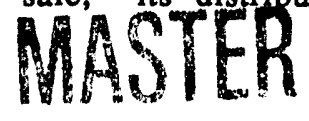




\title{
Decomposition and (Importance) Sampling Techniques for Multi-Stage Stochastic Linear Programs *
}

\author{
Gerd Infanger ${ }^{\dagger}$
}

1993

\begin{abstract}
The difficulty of solving large-scale multi-stage stochastic linear programs arises from the sheer number of scenarios associated with numerous stochastic parameters. The number of scenarios grows exponentially with the number of stages and problems get easily out of hand even for very moderate numbers of stochastic parameters per stage. Our method combines dual (Benders) decomposition with $M$ onte Carlo sampling techniques. We employ importance sampling to efficiently obtain accurate estimates of both expected future costs and gradients and right-hand sides of cuts. The method enables us to solve practical large-scale problems with many stages and numerous stochastic parameters per stage. We discuss the theory of sharing and adjusting cuts between different scenarios in a stage . We derive probabilistic lower and upper bounds, where we use importance path sampling for the upper bound estimation. Initial numerical results turned out to be promising.
\end{abstract}

\footnotetext{
"Research leading to this paper was partially supported by the Office of Naval Research Contract N00014-89-J-1659; the National Science Foundation Grants ECS-8906260, DMS-8913089, the Electric Power Research Institute Contract RP 8010-09, CSA-4005335, and the Austrian Science Foundation, "Fonds zur Förderung der wissenschaftlichen Forschung," Grant J0323-Phy. Any opinions, findings, and conclusions or recommendations expressed in this publication are those of the authors and do NOT necessarily reflect the views of the above sponsors.

†Correspondence: Dr. Gerd Infanger, Department of Operations Research, Stanford University, Stanford, CA 95305-4022, infanger@or.stanford.edu.
} 


\section{Introduction}

The use of stochastic programming techniques for solving large dynamic systems under uncertainty has been hampered until recently by the sheer size of the problems constructed when they are restated as deterministic linear programs. To solve them it has been necessary to keep the number of scenarios representing uncertainties fairly small. Only a few attempts have been made to solve practical multi-stage decision models whose future events are spread over several periods. In this paper we build upon techniques that combine Benders (1962) [3] decomposition and importance sampling (Dantzig and Glynn (1990) [15] and Infanger (1992) [44]) and derive the theory of decomposition and sampling for solving large-scale multi-stage problems.

Stochastic programs with recourse were first introduced independently by Dantzig (1955) [13] and Beale (1955) [2]. Over the years, the bottleneck of stochastic optimization remained to be efficient solution algorithms. Up to recently, the size of stochastic models prohibited their solution, even in the case of considering very modest numbers of uncertain parameters. A number of different algorithmic approaches have been proposed for solving stochastic linear programs. We can broadly categorize these approaches as follows: Methods that attempt to solve the corresponding deterministic equivalent problem directly or by exploiting structure, approximation schemes that attempt to obtain deterministic upper and lower bounds to the problem and consequently refine these bounds iteratively, and sampling based algorithms. Most research activities have concentrated on two-stage stochastic programs and only a few attempts have concerned multi-stage problems.

Exploiting structure, Van Slyke and Wets (1969) [68] showed with their L-shaped method how Benders (1962) [3] decomposition can be applied to solving two-stage stochastic linear programs. Their algorithm uses expected-value cuts, representing an outer linearization of the expected second-stage costs (or the recourse function). A variant proposed later by Birge and Louveaux (1985) [6] is based on multiple cuts, where a different cut with respect to each scenario is computed, and the expectedvalue calculation is carried out in the master problem. Earlier, Dantzig and Madansky (1961) [20] pointed out that the dual of the two-stage stochastic linear program has a structure ideal for Dantzig-Wolfe (1960) [21] decomposition. Birge (1985) [5] extended the L-shaped method to multi-stage stochastic programs, employing a nested Benders decomposition scheme. Gassmann (1990) [34], based on results by Wittrock (1983) [74] for deterministic multi-stage programs, explored different tree-traversing strategies in a Benders decomposition framework for stochastic multi-stage programs. This work has been extended by Morton (1992) [55]. Rockafellar and Wets (1989) [66] presented "Progressive Hedging", in which non-anticipativity constraints are enforced via Lagrangian penalty terms in the objective, and in each iteration a two-stage or multi-stage program is solved for each scenario. It provides an iterative method of solving scenario optimization problems to construct the solution of the stochastic problem. Numerical results of the performance of "Progressive Hedging" have been given by Mulvey and Vladimirou (1989) [57], [58]. "Variable Splitting" formulations 
represent a different way of exploiting structure of a deterministic equivalent program. It makes interior point methods work better on stochastic programs. Lustig et al. (1991) [53] empirically studied the performance of interior-point linear programming solvers on different formulations of two-stage stochastic linear programs. Ruszczynski (1986) [67] proposed his regularized decomposition method.

A classical approximation scheme for solving two-stage stochastic linear programs with stochastic right-hand sides is to calculate upper and lower bounds via the inequalities of Jensen (1906) [45] and Edmundson (1956) [25] and Madansky (1959) [54], respectively, and to successively improve these bounds. Refinements of this bound have been proposed by Ben Tal and Hochman (1972) [4], Kall (1974) [46], Huang, Ziemba and Ben-Tal (1977) [38], Kall and Stoyan (1982) [48], Frauendorfer and Kall (1988) [32], Dupačowá (1978) [24], Gassmann and Ziemba (1986) [35], Frauendorfer (1988) [30] and Birge and Wallace (1988), and Frauendorfer (1992) [31]. Birge and Wets (1987) [9] and Cipra (1985) [12] computed bounds based on solving a generalized moment problem, Birge and Wets (1986) [8] and (1989) [10] exploited the sublinear property of the recourse function, and Birge and Wallace (1988) [7] provided a separable piecewise linear upper bound. Upper bounds for the expectation of convex functions with discrete random variables and the relationship of moment problems and linear programming have been investigated by Prékopa (1988) [63], (1989) [64], and (1990) [65].

Stochastic quasigradient methods select sequentially random search directions based on a limited number of observations of the random function in each iteration. They have been studier by Ermoliev (1983) [26], (1988) [27] [28] and Gaivoronski (1988) [33]. The convergence rates of stochastic quasigradient methods are slow; it is important how well objective values, subgradients, and stepsizes can be specified.

Higle and Sen (1989) [41] developed a "Stochastic Decomposition" Benders Decomposition method, which, like the stochastic quasigradient algorithm, only requires one observation or a very small number of observations per iteration, and which asymptotically creates an outer linearization of the second-stage costs. Optimality conditions and stopping rules for their method have been presented in Higle and Sen (1989) [42].

King and Wets (1989)[49] have applied the theory of epi-consistency in order to obtain consistency results for sequences of optimal solutions, obtained by solving samples of stochastic programming problems .

Monte Carlo methods are known to be efficient for multi-dimensional numerical integration. Efficient probabilistic bounds can be obtained using variance reduction techniques. Pereira et al. (1989) [61] used control variables as a variance-reduction technique in Monte Carlo sampling in a modified Benders decomposition framework. Pereira and Pinto (1991) [62] proposed stochastic dual dynamic programming, a dual Benders decomposition approach, exploiting the piecewise linear property of the recourse function, and introduced path sampling for obtaining estimates of upper bounds. Dantzig and Glynn (1990) [15] and Infanger (1992) [44] used importance 
sampling, based on an additive approximation function, as a variance-reduction technique for Monte Carlo sampling for stochastic linear programs. Krishna (1992) [50] explored in his Ph.D. thesis using piecewise linear approximation functions.

In this paper we use Monte Carlo importance sampling in combination with dual decomposition. We address problems with numerous stochastic parameters, in which the number of possible scenarios per stage is astronomically large and therefore can only be solved using sampling techniques. The paper is organized as follows: We start by introducing a general class of multi-stage stochastic linear programs (Section 2) and a framework for dealing with dependency between stochastic parameters (Section 3). In Section 4 we discuss how dual (Benders) decomposition can be applied to multi-stage problems. Based on that we discuss in Section 5 properties of cuts in the multi-stage framework with the view-point of sharing cuts between different scenarios. Sharing of cuts is crucial to the solution algorithm, as it is a sine qua non condition for employing sampling techniques. The discussion covers the case of inter-stage independence of stochastic parameters as well as cases of inter-stage dependency. In Section 6 we give a brief review of Monte Carlo (importance) sampling as it has been developed it in earlier papers (Dantzig and Glynn (1990) [15] and Infanger (1992) [44]). We then carry on developing probabilistic lower and upper bounds (Section 7 ) for multi-stage problems. In Section 8 we state the algorithn. Initial numerical results are reported in Section 9.

\section{Multi-Stage Stochastic Linear Programs}

In this section we state a general class of multi-stage problems.

Multi-stage planning problems can often be formulated as linear programs with a dynamic matrix structure which, in the deterministic case, appears in a staircase pattern of blocks formed by non-zero sub-matrices. These blocks correspond to and are different for different time periods. In the stochastic case, the blocks of coefficients and right-hand sides in different time periods are functions of several parameters whose values vary stochastically according to dependent and independent distributions which we assume to be known. The resulting problem is a multi-stage stochastic linear program. Even for a problem with a small number of stochastic parameters per stage, the size of the multi-stage problem, when expressed in equivalent deterministic form, can get so large as to appear intractable.

The multi-stage stochastic extension of a deterministic dynamic linear program can be formulated as follows: 


$$
\begin{aligned}
& \min z= \\
& c_{1} x_{1}+E\left(c_{2} x_{2}^{\omega_{2}}+\cdots+E\left(c_{T-1} x_{T-1}^{\omega_{T-1}, \ldots, \omega_{2}}+E\left(c_{T} x_{T}^{\omega_{T}, \ldots, \omega_{2}}\right)\right)\right) \\
& \mathrm{s} / \mathrm{t} \\
& A_{1} x_{1}=b_{1} \\
& -B_{1}^{\omega_{2}} x_{1}+A_{2} x_{2}^{\omega_{2}}+b_{2}^{\omega_{2}} \\
& -B_{T-1}^{\omega_{T}} x_{T-1}^{\omega_{T-1}, \ldots, \omega_{2}}+A_{T} x_{T}^{\omega_{T}, \ldots, \omega_{2}}=\dot{b}_{T}^{\omega_{T}} \\
& x_{1}, \quad x_{2}^{\omega_{2}}, \ldots, \quad x_{T-1}^{\omega_{T-1}, \ldots, \omega_{2}} \quad, \quad x_{T}^{\omega_{T}, \ldots, \omega_{2}} \geq 0 \\
& \omega_{t} \in \Omega_{t}, t=2, \ldots, T \text {. }
\end{aligned}
$$

While the first-stage parameters $c_{1}, A_{1}, b_{1}$ are known to the planner with certainty, the parameters of stages $2, \ldots, T$ are assumed to be known only by their distributions. We consider uncertainty in the coefficients of the transition matrices $B_{t}^{\omega_{t}}, t=1, \ldots, T-1$ and of the right-hand sides $b_{t}^{\omega_{t}}, t=1, \ldots, T$. We assume the coefficients of the technology matrices $A_{t}, t=1, \ldots, T$, and the objective function coefficients $c_{t}, t=2, \ldots, T$, to be known with certainty. The assumption of deterministic technology matrices and objective function coefficients eases the presentation but is not crucial to the solution method which we have developed. The goal of the planner is to minimize the expected value of present and future costs.

In a general model, one has to allow the stochastic parameters of the transition matrices $B_{t}^{\omega_{t}}$ and the right-hand sides $b_{t}^{\omega_{t}}$ to be dependent both within a certain stage and between stages. In the latter case the distributions of the stochastic parameters in period $t$ depend on the outcomes of the stochastic parameters in period $t-1$.

The underlying "wait-and-see" decision-making process is as follows: The decision maker makes a first-stage decision $\hat{x}_{1}$ before observing any outcome of the random parameters. Then he waits until an outcome of the second-stage randum parameters is realized. The second-stage decision is then made based on the knowledge of the realization $\omega_{2}$ but without observing the outcome of any random parameters of stages $3, \ldots, T$, and so forth. As the state (the actual outcome) is carried forward to the following period, the decision tree grows exponentially with the number of stages. The corresponding decision tree is given in Figure 1. The figure represents the example of a four-stage problem with only 3 outcomes per stage.

We consider discrete distributions of random parameters with a finite number of outcomes, e.g., $\omega_{t} \in \Omega_{t}, \Omega_{t}=\left\{1, \ldots, K_{t}\right\}, t=1, \ldots, T$, with corresponding probabilities $p_{t}^{w_{t}}$. With $K_{t}$ being the number of scenarios in period $t$, the total number of scenarios for all $T$ stages is $\prod_{t=1}^{T} K_{t}$. The number $K_{t}$ is expected to be large, as it is derived from crossing the sets of possible outcomes of the different random parameters within a period. For example, if the dimension of the random vector in period $t$ is $h_{t}$ and $\Omega_{t}^{j}$ contains $k_{t}^{j}$ elements, then $K_{t}=\prod_{j=1}^{h_{t}} k_{j}^{t}$. 
For example, consider the case of 20 independent random parameters with 5 outcomes each: the number of scenarios per period is $5^{20} \approx 10^{14}$. If there are only 3 periods, then the total number of scenarios grows to $10^{28}$. These dimensions make it of course impossible to write down the corresponding deterministic equivalent linear program explicitly. See, e.g., Dantzig and Infanger (1991) [17] and (1993) [19] for important application of multi-stage stochastic linear programs in the areas of portfolio optimization and operations planning of hydro-power systems.

\section{Dependency Among Stochastic Parameters}

In the case of independence of the stochastic parameters we define $V_{t}$ to be an $h_{t}$ dimensional random vector, e.g., $V_{t}=\left(V_{1, t}, \ldots, V_{h_{t}, t}\right)$, with $v_{t}^{\omega_{t}}$ (or $\left.v_{t}\right)$ being random outcomes with corresponding probability $p\left(v_{t}^{\omega_{t}}\right)$ (or $\left.p\left(v_{t}\right)\right)$. Because of the independence of the individual random parameters of $V_{t}, p\left(v_{t}\right)=p\left(v_{t}^{1}\right) p\left(v_{t}^{2}\right) \cdots p\left(v_{t}^{h_{t}}\right)$.

We model dependency between stochastic parameters both within a certain stage and between stages. We refer to the former kind of dependency as intra-stage dependency and to the latter as inter-stage dependency.

We define the vector $W_{t}$ to be the vector of all dependent random parameters of stage $t$. $W_{t}$ contains both the random parameters in the transition matrix $B_{t-1}$ and the random parameters of the right-hand side $b_{t}$. We will use the notation

$$
W_{t}=\operatorname{vec}\left(B_{t-1}, b_{t}\right)
$$

to express that all random parameters in the transition matrix $B_{t-1}$ and all random parameters in the right-hand side $b_{t}$ are organized in the random vector $W_{t}$. Each component of $W_{t}$ refers uniquely to an element of the matrix $B_{t-1}$ or to an element of the vector $b_{t}$. We define inverse operations of $W_{t}=\operatorname{vec}\left(B_{t-1}, b_{t}\right)$ as

$$
B_{t-1}\left(W_{t}\right)=\operatorname{vec}_{B}^{-1}\left(W_{t}\right)
$$

and

$$
b_{t}\left(W_{t}\right)=\operatorname{vec}_{b}^{-1}\left(W_{t}\right)
$$

to express that, given the random vector $W_{t}$, the corresponding random elements in $B_{t-1}$ and $b_{t}$ are uniquely determined. With $m_{t+1} \times n_{t}$ being the dimensions of the transition matrices $B_{t}$ and $m_{t}$ being the dimension of the right-hand side $b_{t}$, the dimension of the dependent random vector $W_{t}$ is at most $m_{t+1} \times n_{t}+m_{t+1}$. We denote the dimension of $W_{t}$ with $\tilde{h}_{t}$, thus $W_{t}=\left(W_{1, t}, \ldots, W_{\tilde{h}_{t, t}}\right)$. With respect to the solution procedure discussed later, we formulate a special kind of dependency model which is based on linear intra-stage and inter-stage dependency.

\subsection{Intra-Stage Dependency}

We describe correlation of random parameters within a stage by a linear relation, i.e., outcomes of the $\tilde{h}_{t}$ uncertain dependent parameters $\left(W_{1, t}, \ldots, W_{\tilde{h}_{t}, t}\right)$ can be 
obtained by multiplying outcomes of a vector of independent random parameters $V_{t}=\left(V_{1, t}, \ldots, V_{h_{t}, t}\right)$ by a matrix $\tilde{\beta}_{t}\left(\tilde{h}_{t} \times h_{t}\right)$,

$$
W_{t}=\tilde{\beta}_{t} V_{t}
$$

We denote an outcome of the independent random vector $V_{t}$ by $v_{t}$ or $v_{t}^{\omega_{t}}$, where $\omega_{t} \in \Omega_{t}$. We denote an outcome of an individual random parameter in period $t$, say $V_{t}^{j_{t}}$, by $v_{t}^{j_{t}}$, also denoted as $v^{j_{t}, \omega_{t}}$, where $\omega_{t}^{j_{t}} \in \Omega_{t}^{j_{t}}$, with corresponding probability $p\left(v_{t}^{j_{t}}\right)=$ prob $\left(V_{t}^{j_{t}}=v_{t}^{j_{t}}\right)$. The set of all possible outcomes in stage $t$ is constructed by crossing the sets of possible outcomes of the components of $V_{t}, \Omega_{t}^{j_{t}}, j_{t}=1, \ldots, h_{t}$, as $\Omega_{t}=\Omega_{t}^{1} \times \Omega_{t}^{2} \times \cdots \times \Omega_{t}^{h_{t}}$. Because of the independence of the individual random parameters of $V_{t}, p\left(v_{t}\right)=p\left(v_{t}^{1}\right) p\left(v_{t}^{2}\right) \cdots p\left(v_{t}^{h_{t}}\right)$. The corresponding outcomes of the dependent random vectors $W_{t}$ are denoted by $w_{t}$ or $w_{t}^{\omega_{t}}$.

The linear intra-stage dependency model generates dependent scenarios based on random variations of independent random parameters. This property will become particularly important when implementing sampling for generating scenarios. In many applications the number of independent random parameters per stage, $h_{t}$, is expected to be much smaller than the number of dependent random parameters, $\tilde{h}_{t}$.

\subsection{Inter-Stage Depenć zncy}

For expository purposes we consider an equal number of independent random parameters in each of the different stages and denote this number as $h$. We consider inter-stage dependency as a Markovian process (linear dependency).

$$
w_{t}^{\omega_{t}, \ldots, \omega_{2}}=\beta_{0} v_{t}^{\omega_{t}}+\beta_{1} w_{t-1}^{\omega_{t-1}}+\cdots+\beta_{t-2} w_{2}^{\omega_{2}} .
$$

The matrices $\beta_{0}, \beta_{1}, \ldots, \beta_{T-2}$ are matrices of dimension $(h \times h)$. If they are diagonal matrices the value of an outcome of the random parameter $W_{t}^{i}$ is a weighted sum of some independent random variation in period $t$, denoted by $v_{t}^{i}$, and the values of the observed outcomes in the previous periods $t-1, t-2, \ldots, 2$. If the matrices $\beta_{0}, \beta_{1}, \ldots, \beta_{T-2}$ are non-diagonal matrices, the outcome of each element in $W_{t}$ is dependent upon the outcomes of all elements in $W_{t-1}, W_{t-2}$ back to $W_{2}$ and the independent random variations of $V_{t}$. Given observed outcomes of $W_{t-1}, W_{t-2}$ back to $W_{2}$ one can easily generate a scenario of $W_{t}$ by independent random variations of $V_{t}$. One can see that a scenario in period $t$ is determined based on the observed historical outcomes of the dependent random parameters and by the variations of the independent random parameters in period $t$. We refer to our linear dependency model as an auto-correlative model. The class of auto-correlative models is a wide class of deperdency models that is well studied in the literature. Many different applications in practice can be formulated using the auto-correlative approach. The parameters of the auto-correlative model can be estimated based on historical observations of the outcomes of the stochastic parameters. We can now state the auto-correlative model 
in the notation of the formulation of the multi-stage stochastic linear program:

$$
\begin{gathered}
B_{t-1}\left(w_{t}^{\omega_{t}, \ldots, \omega_{2}}\right)=\operatorname{vec}_{B}^{-1}\left(\beta_{0} v_{t}^{\omega_{t}}\right)+\sum_{\tau=1}^{t-2} \operatorname{vec}_{B}^{-1}\left(\beta_{\tau} \operatorname{vec}\left(B_{t-\tau-1}^{\omega_{t-\tau}}, b_{t-\tau}^{\omega_{t}-\tau}\right)\right) \\
b_{t}\left(w_{t}^{\omega_{t}, \ldots, \omega_{2}}\right)=\operatorname{vec}_{b}^{-1}\left(\beta_{0} v_{t}^{\omega_{t}}\right)+\sum_{\tau=1}^{t-2} \operatorname{vec}_{b}^{-1}\left(\beta_{\tau} \operatorname{vec}\left(B_{t-\tau-1}^{\omega_{t}-\tau}, b_{t-\tau}^{\omega_{t-r}}\right)\right)
\end{gathered}
$$

The general auto-correlative model of equations (3.6) and (3.7) above covers a wide range of models reflecting additive dependency. Special cases, where uncertainty appears either in the right-hand sides $b_{t}$ or in the transition matrices $B_{t-1}$, but not in both at the same time, are common practical examples.

\section{Dual Decomposition}

A description of how the Benders (1962) [3] decomposition algorithm can be applied to solve two-stage stochastic linear programs can be found in Van Slyke and Wets (1969) [68]. Birge (1985) [5] extended the two-stage concept for solving multi-stage stochastic linear programs by using a nested Benders decomposition scheme.

Using dual decomposition we decornpose the problem into subproblems for different stages $t$. In the most general case, in which there is a dependency of stochastic parameters between stages, the number of subproblems is equal to the number of scenarios in each stage $t$. To distinguish one subproblem from another, each is indexed with $\omega_{t}, \ldots, \omega_{2}$, where $\omega_{t}$ is the random event in stage $t$ and $\omega_{t-1}, \ldots, \omega_{2}$ is the path of previous events which gave rise to the particular subproblems in stage $t$.

For expository purposes, we assume initially that the random events occuring in one stage are independent of those that happened in the previous stage. In the independent case, scenarios $\omega_{t+1} \in \Omega_{t+1}$ in period $t+1$ are identical for each scenario $\omega_{t} \in \Omega_{t}$ in period $t$. The history is only carried forward through optimal decisions $\hat{x}_{t-1}^{w_{t}-1, \ldots, \omega_{2}}$ from previous periods.

The idea of using dual decomposition is to express in each stage $t$, and scenario $\omega_{t}$, the expected future costs (the impact of stages $t+1, \ldots, T$ ) by a scalar $\theta_{t}$. The value of $\theta_{t}$ is constrained by a set of "cuts", which are necessary conditions for feasibility and optimality expressed solely in terms of the stage $t$ decision variables $x_{t}$ and $\theta_{t}$. Cuts are initially absent and then sequentially added to the stage $t$ problems. Each scenario subproblem collects the information about expected future costs by means of the cuts.

The relation between the different stages and scenarios in the decomposed multistage problem is summarized as follows: 
The Stage 1 Problem:

$$
\begin{array}{rrr}
\min z_{1}= & c_{1} x_{1}+\theta_{1} & \\
\mathrm{~s} / \mathrm{t} & & \\
\pi_{1}: & A_{1} x_{1} & b_{1} \\
\rho_{1}^{l_{1}}: & -G_{1}^{l_{1}} x_{1}+\alpha_{1}^{l_{1}} \theta_{1} & \geq g_{1}^{l_{1}}, \quad l_{1}=1, \ldots L_{1} \\
& x_{1} & \geq 0 .
\end{array}
$$

The Stage $t, t=2, \ldots, T-1$, Problems:

$$
\begin{aligned}
& \min z_{t}^{\omega_{t}}=c_{t} x_{t}^{\omega_{t}}+\theta_{t}^{\omega_{t}} \\
& s / t
\end{aligned}
$$

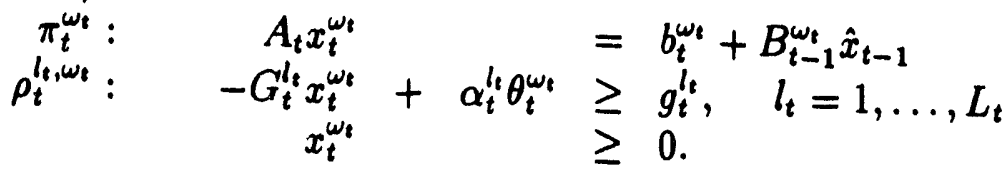

The Stage $T$ Problem:

$$
\begin{aligned}
& \min z_{T}^{\omega_{T}}=c_{T} x_{T}^{\omega_{T}} \\
& s / t \\
& \pi_{T}^{\omega_{T}}: \quad A_{T} x_{T}^{\omega_{T}}=b_{T}^{\omega_{T}}+B_{T-1}^{\omega_{T}} \hat{x}_{T-1} \\
& x_{T}^{\omega_{T}} \geq 0 \text {. }
\end{aligned}
$$

$\min z_{1}$ represents the optimal objective function value in the first stage, and $x_{1}, \theta_{1}$ represent the optimal solution. The vector $\pi_{1}$ denotes the optimal dual prices associated with the original first-stage constraints, and the scalars $\rho_{1}^{t_{1}}$ are the optimal dual prices associated with the cuts which have been added so far in decomposition iterations $l_{1}=1, \ldots, L_{1}$. In later stages, the optimal objective function values $\min z_{t}^{\omega_{t}}=\min z_{t}^{\omega_{t}}\left(\hat{x}_{t-1}\right)$, the optimal dual prices $\pi_{t}^{\omega_{t}}=\pi_{t}^{\omega_{t}}\left(\hat{x}_{t-1}\right)$ associated with the original stage $t$ constraints, and the optimal dual prices $\rho_{t}^{l_{t}, \omega_{t}}=\rho_{t}^{l_{t}, \omega_{t}}\left(\hat{x}_{t-1}\right)$ associated with the cuts are all dependent upon $\hat{x}_{t-1}$, the optimal solution passed as input from the previous stage $t-1$. According to the scenario evolution in the previous stages, an optimal solution $\hat{x}_{t-1}$ is actually indexed by the scenario outcomes of all previous stages and is therefore denoted as $\hat{x}_{t-1}^{\omega_{t-1}, \ldots, \omega_{2}}$. For the sake of exposition, we suppress the scenario history and present the optimal solution of subproblems in stage $t$, scenario $\omega_{z}$ as a function of the input $\hat{x}_{t-1}$.

We compute the expected future costs as

$$
z_{t+1}\left(\hat{x}_{t}^{l_{t}}\right)=E_{\omega_{t+1}} z_{t+1}^{\omega_{t+1}}\left(\hat{x}_{t}^{l_{t}}\right)
$$

the expected right-hand sides of the cuts as

$$
g_{t}^{l_{t}}=E_{\omega_{t+1}}\left(\pi_{t+1}^{\omega_{t+1}}\left(\hat{x}_{t}^{l_{t}}\right) b_{t+1}^{\omega_{t+1}}+\sum_{l_{t+1}=1}^{L_{t+1}} \rho_{t+1}^{l_{t+1}, \omega_{t+1}}\left(\hat{x}_{t}^{l_{t}}\right) g_{t+1}^{l_{t+1}}\right)
$$


and the expected coefficients of the cuts as

$$
G_{t}^{l_{t}}=E_{\omega_{t+1}} \pi_{t+1}^{\omega_{t+1}}\left(\hat{x}_{t}^{l_{t}}\right) B_{t}^{\omega_{t+1}}
$$

where

$$
\rho_{T}^{\omega_{T}}=0, G_{T}^{\omega_{T}}=0, \text { and } g_{T}^{\omega_{T}}=0 .
$$

A subproblem in stage $t$ and in scenario $\omega_{t}$ interacts with its predecessors and descendants by passing cuts backward and optimal solutions forward. Dual decomposition splits the multi-stage problem into a series of two-stage relations which are connected overall by a nesting scheme. We call the stage $t$, scenario $\omega_{t}$ problem the current master problem. It receives from its ancestor in period $t-1$ a solution $\hat{x}_{t-1}$. The current scenario is determined by the outcome $\omega_{t}$ of the random parameters in stage $t$, which are reflected in the right-hand side $b_{t}^{\omega_{t}}+B_{t-1}^{\omega_{t}} \hat{x}_{t-1}$. As stated above, $\hat{x}_{t-1}$ imbeds a history. This history has to be considered when nesting several stages. Given and subject to $\hat{x}_{t-1}$, we solve the stage $t$ problem in scenario $\omega_{t}$ and pass the obtained solution $\hat{x}_{t}^{\omega_{t}}$ to the descendant problems. By solving all problems $\omega_{t+1} \in \Omega_{t+1}$ (referred to as the universe case), we can compute exactly the expected value of the descendant stage costs $z_{t+1}$ and the coefficients $G_{t}^{t_{t}}$ and right-hand side $g_{t}^{l_{t}}$ of a cut. The cut is added to the current master problem (stage $t$, scenario $\omega_{t}$ ), and by solving this problem again, another trial solution is obtained.

The optimal trial solution of the current master problem in stage $t$, scenario $\omega_{t}$ gives a lower bound, and the expected cost of this trial solution gives an upper bound for the expected costs of all scenarios descendant from stage $t$ scenario $\omega_{t}$. If the lower and upper bounds are sufficiently close, the current master problem is deemed to represent adequately the future expected costs and contains (by means of a sufficient number of cuts) all the information needed from future scenarios. In this case we say the current master is balanced with its descendant problems.

Note that the current master problem represents the expected future costs only subject to the trial solution $\hat{x}_{t-1}$, which was passed from its ancestor, and subject to the current scenario $\omega_{t}$. Furthermore we have implicitly assumed that the descendant problems in stage $t+1$ are also balanced with their descendant problems. Note, however, that the solution of the current stage $t$, scenario $\omega_{t}$ problem gives a lower bound for the expected costs of all descendant scenarios regardless of having collected a sufficient number of cuts. We shall exploit this fact.

\section{Properties of the Cuts}

First we discuss various properties of cuts in the case of independence of stochastic parameters between stages. Then we extend the discussion to the case of Markovian dependency and restate the derived properties for the dependent case.

\subsection{The Case of Independence of Stochastic Parameters Between Stages}

The following properties of cuts are crucial for the solution procedure. 
Cuts derived from any trial solution $\hat{x}_{t}^{\omega_{t}}$ are valid in all scenarios $\omega_{t} \in \Omega_{t}$.

The cut $\theta_{t} \geq E_{\omega_{t+1}} \pi_{t+1}^{\omega_{t+1}} B_{t}^{\omega_{t+1}} x_{t}+E_{\omega_{t+1}}\left(\pi_{t+1}^{\omega_{t+1}} b_{t+1}^{\omega_{t+1}}+\sum_{l_{t+1}=1}^{L_{t+1}} \rho_{t+1}^{l_{t+1}, \omega_{t+1}} g_{t+1}^{l_{t+1}}\right)$ is a constraint whose coefficients do not depend on $x_{t}$; hence, it is valid for all values of $x_{t}$. We exhibit this property via the example of cuts generated for the stage $T-1$ master problem and show that these cuts are valid for all values $x_{T-1}^{\omega_{T-1}}$. We employ dual (Benders) decomposition theory to obtain the relation between the stage $T-1$ master problem and the stage $T$ subproblems. First we re-state the stage $T-1$ master problem of the multi-stage stochastic linear program:

The Stage $T-1$ Relaxed Master Problem:

$$
\begin{aligned}
z_{T-1}=\min c_{T-1} x_{T-1}+z_{T}\left(x_{T-1}\right) & \\
\mathrm{s} / \mathrm{t} \quad\left(A_{T-1} x_{T-1}\right. & \left.=b_{T-1}^{w_{T-1}}+B_{T-2}^{\omega_{T-1}} \hat{x}_{T-2}\right) \\
& \geq 0 .
\end{aligned}
$$

System (5.1) represents the relaxed stage $T-1$ master problem, where no cuts have been added so far. As an initial step, we also relax the stage $T-1$ original constraints $\left(A_{T-1} x_{T-1}=b_{T-1}^{\omega_{T-1}}+B_{T-2}^{\omega_{T-1}} \hat{x}_{T-2}\right)$, as indicated by setting them between parentheses. This is to make the stage $T-1$ problem independent of the different scenarios $\omega_{T-1}$ and independent of any history that is represented by $\hat{x}_{T-2}$ passed from the previous stage. Thus we can interpret the stage $T-1$ master problem as being completely cut off from the previous stages. The expected (future) stage $T$ costs, $z_{T}\left(x_{T-1}\right)$, are represented as a function of the stage $T-1$ decision variable $x_{T-1}$.

Instead of stating the primal stage $T$ problem, we state its dual problem. The dual stage $T$ problem in (5.2) includes all $K_{T}$ scenarios $\omega_{T} \in \Omega_{T}$. Note that the stage $T-1$ decision, $\hat{x}_{T-1}$, appears as a given parameter of the stage $T$ subproblem.

The Stage $T$ Dual Subproblem:

$z_{T}\left(\hat{x}_{T-1}\right)=$

$$
\begin{aligned}
& \max p_{T}^{1} \pi_{T}^{1}\left(b_{T}^{1}+B_{T-1}^{1} \hat{x}_{T-1}\right)+\cdots+p_{T}^{K_{T}} \pi_{T}^{K_{T}}\left(b_{T}^{K_{T}}+B_{T-1}^{K_{T}} \hat{x}_{T-1}\right) \\
& s / t \\
& \pi_{T}^{1} A_{T} \\
& \leq c_{T} \\
& \pi_{T}^{K_{T}} A_{T} \quad \leq c_{T} .
\end{aligned}
$$

We assume the stage $T$ dual subproblem to be finite for all values of $\hat{x}_{T-1}$ passed from the previous period. We define

$$
\pi_{T}^{j}:=\left(\pi_{T}^{1, j}, \ldots, \pi_{T}^{K_{T}, j}\right), j=1, \ldots, q_{T}
$$

to be the vertices of the dual feasible region of the stage $T$ subproblem (5.2). We rewrite the stage $T$ subproblem (5.2) by expressing it in terms of the dual vertices. 
By doing so we also can write the minimum expected costs $z_{T}\left(x_{T-1}\right)$ as a function of $x_{T-1}$, rather than having the problem parameterized by $\hat{x}_{T-1}$ :

$$
z_{T}\left(x_{T-1}\right)=\max _{1 \leq j \leq q_{T}} p_{T}^{1} \pi_{T}^{1, j}\left(b_{T}^{1}+B_{T-1}^{1} x_{T-1}\right)+\cdots+p_{T}^{K_{T}} \pi_{T}^{K_{T}, j}\left(b_{T}^{K_{T}}+B_{T-1}^{K_{T}} x_{T-1}\right) .
$$

Using $\theta_{T-1}$ to represent the smallest upper bound for the stage $T$ costs,

$$
\begin{aligned}
& \min \theta_{T-1} \\
& \theta_{T-1} \geq \max _{1 \leq j \leq q T} p_{T}^{1} \pi_{T}^{1, j}\left(b_{T}^{1}+B_{T-1}^{1} x_{T-1}\right)+\cdots+p_{T}^{K_{T}} \pi_{T}^{K_{T}, j}\left(b_{T}^{K_{T}}+B_{T-1}^{K_{T}} x_{T-1}\right),
\end{aligned}
$$

we car: state the stage $T-1$ master problem as a full master problem with all possible cuts added.

The Stage $T$ - 1 Full Master Problem:

$$
\begin{aligned}
& \min z_{T-1}=c_{T-1} x_{T-1}+\theta_{T-1} \\
& \mathrm{~s} / \mathrm{t}\left(A_{T-1} x_{T-1} \quad=b_{T-1}^{\omega T-1}+B_{T-2}^{\omega_{T-1}} \hat{x}_{T-2}\right) \\
& x_{T-1} \geq 0 \\
& \theta_{T-1} \geq p_{T}^{1} \pi_{T}^{1, j}\left(b_{T}^{1}+B_{T-1}^{1} x_{T-1}\right)+\cdots+p_{T}^{K_{T}} \pi_{T}^{K_{T}, j}\left(b_{T}^{K_{T}}+B_{T-1}^{K_{T}} x_{T-1}\right) \quad j=1, \ldots, q_{T} .
\end{aligned}
$$

In the full master representation for stage $T-1$, each cut corresponds to a vertex of the dual feasible region of the stage $T$ subproblem. The set of all cuts represents a complete outer linearization of the expected stage $T$ costs as a function of $x_{T-1}$. Each cut is valid for each value of $x_{T-1}$ because it is derived from a dual feasible price vector, one of the dual vertices $\pi_{T}^{j}, j=1, \ldots, q_{T}$. Taking now the stage $T-1$ constraints $\left(A_{T-1} x_{T-1}=b_{T-1}^{\omega T}+B_{T-2}^{\omega T-1} \hat{x}_{T-2}\right)$ into consideration, we see that the piecewise-linear representation of the expected stage $T$ costs remains unaffected by the stage $T-1$ constraints. The stage $T-1$ constraints have different right-hand sides in each scenario $\omega_{T-1}$ and for each value of $\hat{x}_{T-2}$ passed from the previous period. Therefore they represent a different set of feasible solutions for $x_{T-1}$ in each scenario $\omega_{T-1}$ and for each value of $\hat{x}_{T-2}$. The piecewise-linear representation of the stage $T$ expected costs by means of the cuts is valid for any scenario $\omega_{T-1}$ and any value of $\hat{x}_{T-2}$ passed from the previous period. Of course, different cuts will be binding in different scenarios $\omega_{T-1}$. For example, a cut can be a support of the recourse function, the minimum expected costs $z_{T}\left(x_{T-1}\right)$ as a function of the stage $T-1$ decision variables $x_{T-1}$, in a particular scenario and may be way below the recourse function in another scenario.

Having stated the full master problem in stage $T-1$ in equation (5.6 - 5.7), we now extend the argument for all stages. Analogously to before, we state the dual problems in stages $t$, where $t=2, \ldots, T-1$. In order to ease the following presentation, we will use the matrix $G_{t}$ to represent the matrix of coefficients of all cuts $G_{t}^{l_{t}}, l_{t}=1, \ldots, L_{t}$, where each $G_{t}^{l_{t}}$ is a row of $G_{t}$, and the column vector $g_{t}$ to represent the right-hand sides of the cuts $g_{t}^{l_{t}}, l_{t}=1, \ldots, L_{t}$. Similarly we define as 


\section{Inter-stage dependency in three-stage models.}

We pointed out before that, in order to be able to share cuts in stage $t-1$ for different scenarios $\omega_{t-1}$, given a history $\omega_{t-2}, \ldots, \omega_{2}$, the stage $t$ dual variables $\pi_{t}^{\omega_{t}, \ldots, \omega_{2}}$ have to be feasible for any history $\omega_{t-3}, \ldots, \omega_{2}$. This property is satisfied in the case discussed above, in which the stochastic parameters of the transition matrices $B_{t-1}^{\omega_{t-1}}$ are interstage independent. Similarly, the property of dual feasibility is retained if we restrict the number of stages of the multi-stage model to $T=3$. In this case, the stage 3 , $\omega_{3}$ subproblems do not contain any cuts (as there are no further stages); therefore, the stage 3 dual feasible region is not affected by any historical outcome $\omega_{2}$. For the 3-stage model we can then formulate the general auto-correlative model, with inter-stage dependency of the transition matrices as well as the right-hand side.

The formulation of the second-stage cuts takes on the form:

$$
\theta_{2} \geq E_{\omega_{3}}\left(\pi_{3}^{\omega_{3}, \omega_{2}}\left(b_{3}^{\omega_{3}, \omega_{2}}+B_{2}^{\omega_{3}, \omega_{2}} x_{2}\right)\right. \text {, }
$$

and substituting the auto-correlative model leads to:

$$
\begin{aligned}
& \theta_{2} \geq \\
& E_{\omega_{3}} \pi_{3}^{\omega_{3}, \omega_{2}}\left(\operatorname{vec}_{b}^{-1}\left(\beta_{0} v^{\omega_{3}}\right)\right)+E_{\omega_{3}} \pi_{3}^{\omega_{3}, \omega_{2}}\left(\operatorname{vec}_{B}^{-1}\left(\beta_{0} v^{\omega_{3}}\right) x_{2}\right)+ \\
& {\left[( E _ { \omega _ { 3 } } \pi _ { 3 } ^ { \omega _ { 3 } , \omega _ { 2 } } ) \left(\operatorname{vec}_{b}^{-1}\left(\beta_{1} \operatorname{vec}\left(B_{1}^{\omega_{2}}, b_{2}^{\omega_{2}}\right)\right)+\right.\right.} \\
& \left.\left(E_{\omega_{3}} \pi_{3}^{\omega_{3}, \omega_{2}}\right)\left(\operatorname{vec}_{B}^{-1}\left(\beta_{1} \operatorname{vec}\left(B_{1}^{\omega_{2}}, b_{2}^{\omega_{2}}\right)\right) x_{2}\right)\right] .
\end{aligned}
$$

Like before, we drop index $\omega_{2}$ as the property of dual feasibility in stage 3 is independent of scenario $\omega_{2}$, and denote the dual variables only as $\pi_{t}^{\omega_{3}}$, and restate the cut as follows:

$$
\begin{aligned}
& \theta_{2} \geq \\
& E_{\omega_{3}} \pi_{3}^{\omega_{3}}\left(\operatorname{vec}_{b}^{-1}\left(\beta_{0} v^{\omega_{3}}\right)\right)+E_{\omega_{3}} \pi_{3}^{\omega_{3}}\left(\operatorname{vec}_{B}^{-1}\left(\beta_{0} v^{\omega_{3}}\right) x_{2}\right)+ \\
& {\left[( E _ { \omega _ { 3 } } \pi _ { 3 } ^ { \omega _ { 3 } } ) \left(\operatorname{vec}_{b}^{-1}\left(\beta_{1} \operatorname{vec}\left(B_{1}^{\omega_{2}}, b_{2}^{\omega_{2}}\right)\right)+\right.\right.} \\
& \left.\left(E_{\omega_{3}} \pi_{3}^{\omega_{3}}\right)\left(\operatorname{vec}_{B}^{-1}\left(\beta_{1} \operatorname{vec}\left(B_{1}^{\omega_{2}}, b_{2}^{\omega_{2}}\right)\right) x_{2}\right)\right] .
\end{aligned}
$$

Compared to the formulation for the general multi-stage problem, the third part of the cut formulation has vanished, and two parts, the first depending on $\omega_{3}$ and the second depending on $\omega_{2}$, remain. The cut can be adjusted to the current scenario $\omega_{2}$ if the expectation $\left(E_{\omega_{3}} \pi_{t}^{\omega_{3}}\right)$ is known. In order to adjust the cut to a given scenario $\omega_{2}$, we specially compute the second and $\omega_{2}$-dependent part of the cut formulation. The first part remains the same for each scenario $\omega_{2}$. We store the calculation and adjust the cut for a given scenario by adding part two using $\left(E_{\omega_{3}} \pi_{3}^{\omega_{3}}\right)$, and update the gradients as well as the right-hand side of the cut.

For three-stage models with auto-correlative dependency, cuts in stage 2 can be adjusted to be valid in any scenario $\omega_{2} \in \Omega_{2}$.

We further demonstrate how to update cuts in the second stage with a simplified example of the general auto-correlative model of equations (5.24) and (5.25). We 
assume a dependency structure in which uncertainty occurs only in the transition matrix, and consider, in order to ease the presentation, instead of matrices $\beta_{\tau}, \tau=0,1$, scalars $\beta_{\tau}^{B}, \tau=0,1$ :

$$
\begin{gathered}
B_{2}^{\omega_{3}}=\beta_{0}^{B} \tilde{\eta}_{3}^{\omega_{3}}+\beta_{1}^{B} B_{1}^{\omega_{2}} \\
B_{1}^{\omega_{2}}=\beta_{0}^{B} \tilde{\eta}_{2}^{\omega_{2}},
\end{gathered}
$$

where for $t=2,3, \tilde{\eta}_{t}^{\omega_{t}}$ is a matrix (of the dimension of $B_{t-1}$ ) of independent random parameters whose elements are functions of random parameters that are independent of the random parameters of previous periods. The corresponding cut,

$$
\theta_{2} \geq E_{\omega_{3}} \pi_{3}^{\omega_{3}}\left(b_{3}+\beta_{0}^{B} \tilde{\eta}_{3} x_{t-1}\right)+\left(E_{\omega_{3}} \pi_{t}^{\omega_{3}}\right) \beta_{1}^{B} B_{1}^{\omega_{2}} x_{t-1},
$$

needs to be adjusted in its coefficients by specializing the term $\left(E_{\omega_{3}} \pi_{t}^{\omega_{3}}\right) \beta_{1}^{B} B_{1}^{\omega_{2}}$ to the stage $2, \omega_{2}$ scenario subproblem which is to be solved.

\subsection{Summary of properties of cuts}

Taking advantage of the above stated properties, we actually only need to store one subproblem per stage $t$. For different scenarios $\omega_{t}$ and different solutions $\hat{x}_{t-1}$ passed from the previous stage, we determine the right-hand side of the subproblem accordingly. Cuts generated by stage $t+1$ subproblems are valid for all scenarios $\omega_{t} \in$ $\Omega_{t}$ in the case of independence of the stochastic parameters between stages. In the case of auto-correlative inter-stage dependency, certain special cases, as described above, allow for "sharing" of valid cuts among scenarios $\omega_{t}$ by reusing common expected dual prices from stage $t+1$ and adjusting applied cuts according to the scenario history up to stage $t, \omega_{t}, \ldots, \omega_{2}$. Future information is represented in the cuts which have been generated so far and can be used in any scenario $\omega_{t} \in \Omega_{t}$ independently of which scenario originated the cut.

\section{Review: Monte Carlo (Importance) Sampling}

The difficulty of solving large-scale stochastic problems arises from the need to compute multiple integrals or multiple sums. Even for a small number of stochastic parameters, say, e.g., 20,the number of terms in the multiple sum computation gets easily out of hand and the problem is no longer practical to solve by direct summation. This is especially true because function evaluations can be computationally expensive since each term in the multiple sum requires the solution of a linear program. Monte Carlo Methods are recommended to compute multiple integrals or multiple sums for higher dimensional sample spaces (Davis and Rabinowitz (1984) [22], Glynn and Iglehart (1989) [37]). We now give a brief review of our Monte Carlo importance sampling scheme (Dantzig and Glynn (1990) [15] and Infanger (1992) [44]) that we have successfully engaged for solving two-stage problems. It allows us to obtain very accurate estimates using only a small sample size. It is therefore a crucial part of the 
solution techniques for multi-stage problems. In order to ease the presentation we supress in this review the stage index $t$.

Suppose $C^{\omega}=C\left(v^{\omega}\right)$ are independent random variates of $v^{\omega}, \omega=1, \ldots, n$ with expectation $z$, where $n$ is the sample size. An unbiased estimator of $z$ with variance $\sigma_{\bar{z}}^{2}=\sigma^{2} / n, \sigma^{2}=\operatorname{var}(C(V))$ is

$$
\bar{z}=(1 / n) \sum_{\omega=1}^{n} C^{\omega}
$$

Note that the standard error decreases with $n^{-0.5}$ and the convergence rate of $\bar{z}$ to $z$ is independent of the dimension of the sample space $h$. We rewrite $z=\sum_{\omega \in \Omega} C\left(v^{\omega}\right) p\left(v^{\omega}\right)$ as

$$
\sum_{\omega \in \mathbb{Z}} \frac{C\left(v^{\omega}\right) p\left(v^{\omega}\right) q\left(v^{\omega}\right)}{q\left(v^{\omega}\right)}
$$

by introducing a new probability mass function $q\left(v^{w}\right)$ and we obtain a new estimator of $z$

$$
\bar{z}=\frac{1}{n} \sum_{\omega=1}^{n} \frac{C\left(v^{\omega}\right) p\left(v^{\omega}\right)}{q\left(v^{\omega}\right)}
$$

by sampling from $q\left(v^{\omega}\right)$. The variance of $\bar{z}$ is given by

$$
\operatorname{var}(\bar{z})=\frac{1}{n} \sum_{\omega \in \Omega}\left(\frac{C\left(v^{\omega}\right) p\left(v^{\omega}\right)}{q\left(v^{\omega}\right)}-z\right)^{2} q\left(v^{\omega}\right) .
$$

Chosing $q^{*}\left(v^{\omega}\right)=C\left(v^{\omega}\right) p\left(v^{\omega}\right) / \sum_{\omega \in \Omega} C\left(v^{\omega}\right) p\left(v^{\omega}\right)$ would lead to $\operatorname{var}(\bar{z})=0$, which means one could get a perfect estimate of the multiple sum from only one estimation. Practically however, this is useless since to compute $q\left(v^{\omega}\right)$ we have to know $z=$ $\sum_{\omega \in \Omega} C^{\omega} p\left(v^{\omega}\right)$, which is the value we wanted to compute in the first place. The result, however, helps us to derive a heuristic criterion for choosing a "good" $q$. It should be proportional to the product $C\left(v^{\omega}\right) p\left(v^{\omega}\right)$ and should have a form that can be integrated easily. Thus a function $\Gamma\left(v^{\omega}\right) \approx C\left(v^{\omega}\right)$ is sought, which can be integrated with less costs than $C\left(v^{\omega}\right)$. Additive and multiplicative (in the components of the stochastic vector $v$ ) approximation functions and combinations of these are potential candidates for our approximations. In particular, we have been getting good results using $C(V) \approx \sum_{i=1}^{h} C_{i}\left(V_{i}\right)$. We compute $q$ as

$$
q\left(v^{\omega}\right) \approx \frac{C\left(v^{\omega}\right) p\left(v^{\omega}\right)}{\sum_{i=1}^{h} \sum_{\omega \in \Omega_{1}} C_{i}\left(v^{\omega}\right) p_{i}\left(v^{\omega}\right)} .
$$

If the additive function turns out to be a bad approximation of the cost function, as indicated by the observed variance being too high, it is easily corrected by increasing the size of the sample. This is done adaptively. 
Actually we use a variant of the additive approximation function. By introducing $C(\tau)$, the costs of a base case, we make the model more sensitive to the impact of the stochastic parameters $v$. Our approximation function is computed as follows:

$$
\Gamma(V)=C(\tau)+\sum_{i=1}^{h} \Gamma_{i}\left(V_{i}\right), \Gamma_{i}\left(V_{i}\right)=C\left(\tau_{1}, \ldots, \tau_{i-1}, V_{i}, \tau_{i+1}, \ldots, \tau_{h}\right)-C(\tau) .
$$

We refer to this as a marginal cost approximation. We explore the cost function at the margins, e.g. we vary the random elements $v_{i}$ to compute the costs for all outcomes $v_{i}$ while we fix the other random elements at the level of the base case. $\tau$ can be any arbitrary chosen point of the set of $k_{i}$ discrete values of $v_{i}, i=1, \ldots, h$. For example we choose $\tau_{i}$ as that outcome of $V_{i}$ which leads to the lowest costs, ceteris paribus.

Summarizing, the importance sampling scheme has two phases: the preparation phase and the sample phase. In the preparation phase we explore the cost function $C(V)$ at the margins to compute the additive approximation function $\Gamma(V)$. For this process $n_{\text {prep }}=1+\sum_{i=1}^{h}\left(k_{i}-1\right)$ subproblems have to be solved. Using $\Gamma(V)$ we compute the approximate importance density

$$
q\left(v^{\omega}\right)=\frac{\Gamma\left(v^{\omega}\right) p\left(v^{\omega}\right)}{C(\tau)+\sum_{i=1}^{h} \sum_{\omega \in \Omega_{i}} \Gamma_{i}\left(v^{\omega}\right) p\left(v^{\omega}\right)}
$$

Next we sample $n$ scenarios from the importance density and, in the sample phase, solve $n$ linear progiams to compute the estimation of $\bar{z}$ using the Monte Carlo estimator. We compute the gradient $G$ and the right hand side $g$ of the cut using the same sample points at hand from the expected cost calculation. See Infanger (1992) [44] for the computation of the cuts and details of the estimation process.

The additive adaptive importance sampling scheme worked very well in many different kinds of models. See Infanger (1992) [44] and Dantzig and Infanger (1991) [18] for numerical results of solving large-scale two-stage stochastic linear programs.

\section{Probabilistic Bounds}

\subsection{Estimates of Expected Values}

For calculating the expected values of the future costs, the gradients, and the righthand sides of the cuts in each stage $t$, we use Monte Carlo importance sampling as discussed in Section 6 for two-stage stochastic linear programs. Employing Monte Carlo sampling techniques means not solving all problems $\omega_{t+1} \in \Omega_{t+1}$ but solving problems $\omega_{t+1} \in S_{t+1}$, where $S_{t+1}$ is a subset of $\Omega_{t+1}$. Instead of the exact expected values $z_{t+1}\left(\hat{x}_{t}\right), G_{t}\left(\hat{x}_{t}\right), g_{t}\left(\hat{x}_{t}\right)$, we compute estimates $\bar{z}_{t+1}\left(\hat{x}_{t}\right), \bar{G}_{t}\left(\hat{x}_{t}\right), \bar{g}_{t}\left(\hat{x}_{t}\right)$ using the importance sampling procedure. We also estimate the error in the estimation of $z_{t+1}\left(\hat{x}_{t}\right)$ by the variance

$$
\sigma_{\bar{z}_{t+1}}^{2}\left(\hat{x}_{t}\right):=\operatorname{var}\left(\bar{z}_{t+1}\left(\hat{x}_{t}\right)\right)
$$


Thus, given a particular $\hat{x}_{t}$, we obtain from the importance sampling procedure an estimate of the mean of the stage $t+1$ costs and the associated error distribution. For sample sizes larger than 40 (see, for example, Davis and Rabinowitz (1984) [22]), one can assume that the error of the estimation is normally distributed. Therefore, we define the estimate of the expected stage $t+1$ costs $\tilde{z}_{t+1}\left(\hat{x}_{t}\right)$ for given $\hat{x}_{t}$ to be a random parameter, normally distributed with mean $\bar{z}_{t+1}\left(\hat{x}_{t}\right)$ and variance $\sigma_{\bar{z}_{t+1}}^{2}$ :

$$
\tilde{z}_{t+1}\left(\hat{x}_{t}\right):=N\left(\bar{z}_{t+1}\left(\hat{x}_{t}\right), \sigma_{\bar{z}_{t+1}}^{2}\left(\hat{x}_{t}\right)\right) \text {. }
$$

A cut with estimated coefficients and right-hand sides differs from a cut obtained by computing the expected values of the coefficients and right-hand sides exactly. The outer linearization

$$
L_{t}\left(\hat{x}_{t}, x_{t}\right)=G_{t}\left(\hat{x}_{t}\right) x_{t}+g_{t}\left(\hat{x}_{t}\right)
$$

with respect to the universe case and

$$
\bar{L}_{t}\left(\hat{x}_{t}, x_{t}\right)=\bar{G}_{t}\left(\hat{x}_{t}\right) x_{i}+\bar{g}_{t}\left(\hat{x}_{t}\right)
$$

with respect to the estimation differ in the gradient and in the right-hand side. We denote as

$$
\epsilon_{t, \hat{x}_{t}}\left(x_{t}\right)=L_{t}\left(\hat{x}_{t}, x_{t}\right)-\bar{L}_{t}\left(\hat{x}_{t}, x_{t}\right)
$$

the difference between between the true (universe) and the estimated value of the outer linearization. At $x_{t}=\hat{x}_{t}$, the value at which the cut was derived, $L_{t}\left(\hat{x}_{t}, \hat{x}_{t}\right)=$ $z_{i+1}\left(\hat{x}_{t}\right)$ and $\bar{L}_{t}\left(\hat{x}_{t}, \hat{x}_{t}\right)=\bar{z}_{t+1}\left(\hat{x}_{t}\right)$. Thus, if a true cut obtained by solving the universe case is binding at the solution $x_{t}=\hat{x}_{t}$, the variable $\theta_{t}$ takes on the value

$$
\theta_{t}=L_{t}\left(\hat{x}_{t}, \hat{x}_{t}\right)=z_{t+1}\left(\hat{x}_{t}\right)
$$

In the case of using Monte Carlo sampling, we relate $\theta_{t}$ to the estimated value of the expected stage $t+1$ costs at $\hat{x}_{t}, \bar{L}_{t}\left(\hat{x}_{t}, \hat{x}_{t}\right)=\bar{z}_{t+1}\left(\hat{x}_{t}\right)$, and correct for estimation error by adjusting the right-hand side. Thus we can state

$$
\begin{gathered}
\theta_{t}=\bar{L}_{t}\left(\hat{x}_{t}, \hat{x}_{t}\right)-\tilde{z}_{t+1}\left(\hat{x}_{t}\right)+z_{t+1}\left(\hat{x}_{t}\right), \\
\theta_{t}=\bar{L}_{t}\left(\hat{x}_{t}, \hat{x}_{t}\right)+\epsilon_{t, \hat{x}_{t}}\left(\hat{x}_{t}\right) .
\end{gathered}
$$

Equation (7.7) represents a valid statement for a solution $x_{t}=\hat{x}_{t}$. The correction term $\epsilon_{t, \hat{x}_{t}}\left(\hat{x}_{t}\right)=z_{t+1}\left(\hat{x}_{t}\right)-\bar{z}_{t+1}\left(\hat{x}_{t}\right)$ corrects for the estimation error. Of course, we do not know the difference $z_{t+1}\left(\hat{x}_{t}\right)-\bar{z}_{t+1}\left(\hat{x}_{t}\right)$ explicitly for each cut when we compute it. However, we can obtain an estimate of the distribution of the correction term from the estimation process. Recall that by using Monte Carlo importance sampling, we obtain an unbiased estimate of $z_{t+1}\left(\hat{x}_{t}\right), \bar{z}_{t+1}\left(\hat{x}_{t}\right)$, with variance $\sigma_{\bar{t}_{t+1}}^{2}\left(\hat{x}_{t}\right)$. Therefore, $\epsilon_{t, \hat{x}_{t}}\left(\hat{x}_{t}\right)$ is normally distributed with mean 0 and variance $\left.\sigma_{\hat{z}_{t+1}}^{2}\left(\hat{x}_{t}\right)\right)$ :

$$
\epsilon_{t, \hat{x}_{t}}\left(\hat{x}_{t}\right):=N\left(0, \sigma_{\bar{z}_{t+1}}^{2}\left(\hat{x}_{t}\right)\right) .
$$


Suppose that a cut $\bar{L}_{t}\left(\hat{x}_{t}, x_{t}\right)=\bar{G}_{t}(\hat{x}) x+\bar{g}(\hat{x})$, computed at $x_{t}=\hat{x}_{t}$, is binding at a solution $\hat{\hat{x}}_{t}$ where $\hat{x}_{t} \neq \hat{x}_{t}$. Applying again a correction for the estimation error we obtain:

$$
\theta_{t}=\bar{L}_{t}\left(\hat{x}_{t}, \hat{\hat{x}}_{t}\right)+\epsilon_{t, \hat{x}_{t}}\left(\hat{\hat{x}}_{t}\right) \text {. }
$$

The correction term for the estimation error is clearly the true value of the cut at $x_{t}=\hat{\hat{x}}_{t}$ minus the value obtained by the sampling procedure:

$$
\epsilon_{t, \dot{x}_{t}}(\hat{\hat{x}})=L_{t}\left(\hat{x}_{t}, \hat{\hat{x}}\right)-\bar{L}_{t}\left(\hat{x}_{t}, \hat{\hat{x}}\right) \text {. }
$$

We do not know the difference $L_{t}\left(\hat{x}_{t}, \hat{\hat{x}}\right)-\bar{L}_{t}\left(\hat{x}_{t}, \hat{\hat{x}}\right)$ when we compute the cut. The distribution of the estimation error, $\epsilon_{t, \hat{x}_{t}}(\hat{\hat{x}})$ at $x_{t}=\hat{\hat{x}}_{t}$, can be computed based on the observations $G_{t}^{\omega_{t}}$ and $g_{t}^{\omega_{t}}$. We will explicit this in the next section.

However, for most practical problems it seems to be a sufficiently close approximation to assume that

$$
\epsilon_{t, \hat{x}_{t}}\left(\hat{\hat{x}}_{t}\right) \approx \epsilon_{t, \hat{x}_{t}}\left(\hat{x}_{t}\right) \text { for } \hat{\hat{x}}_{t} \approx \hat{x}_{t}
$$

This means that we assume the error distribution $\epsilon_{t, \hat{x}_{t}}\left(x_{t}\right)$ to be constant with respect to $x_{t}$, and use $\epsilon_{t, \hat{x}_{t}}\left(\hat{x}_{t}\right)$, rather than taking into account correctly $\epsilon_{t, \hat{x}_{t}}\left(x_{t}\right)$ as a function of $x_{t}$. Ever, thing that will be derived using the constant error term $\epsilon_{t, \hat{x}_{t}}\left(\hat{x}_{t}\right)$ can be extended to use the variable error term $\epsilon_{t, \dot{x}_{t}}\left(x_{t}\right)$. We will show this at the relevant places in the text.

We will in the following denote the constant error term $\epsilon_{t, \hat{x}_{t}}\left(\hat{x}_{t}\right)$ as $\epsilon_{t}$ or as $\epsilon_{t}^{t_{t}}$, short for $\epsilon_{t, \hat{x}_{t}^{l_{t}}}\left(\hat{x}_{t}^{l_{t}}\right)$, when we refer to it in a particular iteration $l_{t}$ of the Benders decomposition algorithm. We will denote the error term as a function of $x_{t}, \epsilon_{t, \hat{x}_{t}}\left(x_{t}\right)$ as $\epsilon_{t}\left(x_{t}\right)$ or as $\epsilon_{t}^{l_{t}}\left(x_{t}\right)$, short for $\epsilon_{t, x_{t}^{l_{t}}}\left(x_{t}\right)$, when referring to it in a particular Benders iteration $l$.

\subsection{The Estimation Error of a Cut}

In order to not blur the view by unneccesarily complicated notation we suppress in the following derivation the stage index $t$.

Using Monte Carlo sampling we estimate the coefficients $G_{i}, i=1, \ldots, n$, and the right-hand side $g$ and obtain $\bar{G}$ and $\bar{g}$ by averaging over $G^{\omega}=G_{1}^{\omega}, \ldots, G_{n}^{\omega}$ and $g^{\omega}$. For example, in the case of crude Monte Carlo sampling, a sample of size $N$ would be obtained by sampling $G^{\omega}, g^{\omega}, \omega=1, \ldots, N$, from the original distribution $p(\omega)$, $\omega \in \Omega$, and an estimate of $G$ and $g$ would be computed by the sample means $\bar{G}, \bar{g}$. In the case of importance sampling, a different and more complicated weighting scheme is used. We do not discuss this here and continue with the case of crude Monte Carlo.

For each sample $\omega, \omega=1, \ldots, N$, we obtain independent observations of the mutually dependent coefficients and right-hand side of a cut:

$$
G_{1}^{\omega}, \ldots, G_{n}^{\omega}, g^{\omega}
$$


Let us denote by $R$ the $n+1 \times N$ matrix composed of the observations of the coefficients and the right-hand side of the cuts, adjusted by the mean of the observations:

$$
R=\left(\begin{array}{ccc}
G_{1}^{1}-\bar{G}_{1} & \cdots & G_{1}^{N}-\bar{G}_{1} \\
\vdots & & \vdots \\
G_{n}^{1}-\bar{G}_{n} & \cdots & G_{n}^{N}-\bar{G}_{n} \\
g^{1}-\bar{g} & \cdots & g^{N}-\bar{g}
\end{array}\right)
$$

An estimate of the variance of the mean value of $\bar{L}(\hat{x}, x)=\bar{G}(\hat{x}) x+\bar{g}(\hat{x})$ can be obtained by

$$
\operatorname{var}(\bar{L}(\hat{x}, x))=\frac{1}{N} \frac{1}{N-1}\left(x^{T}, 1\right) R R^{T}\left(x^{T}, 1\right)^{T} .
$$

Defining

$$
u^{\omega}=R^{\omega}\left(x^{T}, 1\right)^{T}, \omega=1, \ldots, N
$$

where the row vector $R^{\omega}$ extracts the column in $R$ that represents the observation $\omega$,

$$
R^{\omega}=\left(G_{1}^{\omega}-\bar{G}_{1}, \ldots, G_{n}^{\omega}-\bar{G}_{n}, g^{\omega}-\bar{g}\right),
$$

we can write the estimated variance of the value of the cut at $x$ as

$$
\operatorname{var}(\bar{L}(\hat{x}, x))=\frac{1}{N} \frac{1}{N-1} \sum_{\omega=1}^{N}\left(u^{\omega}\right)^{2} .
$$

This estimate can be easily computed. It follows that, with knowledge of the matrix $R$ of observations of the coefficients and the right-hand side of a cut, we are able to compute an estimate of the variance of the value of the cut, $\operatorname{var}(\bar{L}(\hat{x}, x))$, for any value of $x$. Clearly,

$$
\operatorname{var}(\bar{L}(\hat{x}, \hat{x}))=\operatorname{var}(\bar{z}(\hat{x})) .
$$

Using the estimation procedure we obtain an estimate of the distribution of the correction term:

$$
\epsilon_{\hat{x}}(x)=N(0, \operatorname{var}(\bar{L}(\hat{x}, x))
$$

where we also denote

$$
\sigma_{L}^{2}(x):=\operatorname{var}(\bar{L}(\hat{x}, x)) .
$$

The error term $\epsilon_{\hat{x}}(x)$ now correctly represents an estimate of the distribution of the difference $L(\hat{x}, x)-\bar{L}(\hat{x}, x)$ as a function of $x$.

While it seems to be impractical to store the matrix $R$ to obtain the error estimate of each cut as a function of a particular solution of the master problem at which the cut is binding, a Taylor approximation of $\operatorname{var}(\bar{L}(\hat{x}, x))$ seems to be computationally advantageous.

Let $\alpha(\hat{x})$ be the vector of local derivatives at $\hat{x}$ :

$$
\alpha(\hat{x}):=\left[\frac{\partial}{\partial x}(\operatorname{var}(\bar{L}(\hat{x}, x)))\right]_{\hat{x}},
$$


where

$$
\frac{\partial}{\partial x_{i}}(\operatorname{var}(\bar{L}(\hat{x}, x)))=\frac{\partial}{\partial x_{i}}\left(\frac{1}{N} \frac{1}{N-1} \sum_{\omega=1}^{N}\left(u^{\omega}\right)^{2}\right)=\frac{2}{N(N-1)} \sum_{\omega=1}^{N} R^{\omega}\left(x^{T}, 1\right)^{T} R_{i}^{\omega} .
$$

Using a first order Taylor approximation we can write $\operatorname{var}(\bar{L}(\hat{x}, x))$ in the following form:

$$
\begin{aligned}
\sigma_{L}^{2}(x)=\operatorname{var}(\bar{L}(\hat{x}, x)) & \approx \operatorname{var}(\bar{L}(\hat{x}, \hat{x}))+\alpha(\hat{x})(x-\hat{x}) \\
& =\operatorname{var}(\bar{z}(\hat{x}))+\alpha(\hat{x})(x-\hat{x}) .
\end{aligned}
$$

The estimate of the variance $\operatorname{var}(\bar{L}(\hat{x}, x))$ as a function of $x$ is represented as a sum of two terms, the estimated variance of the second-stage costs at $\hat{x}, \operatorname{var}(\bar{z}(\hat{x}))$, plus the linear term that represents the change of the variance, for $x \neq \hat{x}$. The latter term is assumed to be small, if $\alpha(\hat{x})$ is assumed to be small.

\subsection{The Lower Bound Estimate}

In each stage $t, t=2, \ldots, T-1$, a lower bound for the stage $t$ expected costs (subject to scenario $\omega_{t}$ and the solution $\hat{x}_{t-1}$ passed from the previous stage) is represented by the optimal objective function value of the stage $t$ master problem, $z_{t}^{\omega_{t}}$. In each stage $t, t=2, \ldots, T-1$, the cuts added so far represent an outer linearization of the expected future costs. For an optimal solution $\left(\hat{x}_{t}, \hat{\theta}_{t}\right)$ of the stage $t$ master problem, $\hat{\theta}_{t}$ represents a lower bound for the value of the expected future costs. A lower bound for the total expected costs of the multi-stage stochastic linear program is represented by the optimal objective function value of the stage 1 master problem, $z_{1}$, which includes a value for $\hat{\theta}_{1}$ representing a lower bound for the value of the expected future costs (in stages $2, \ldots, T)$. If cuts have been obtained in stage $t$ by solving the stage $t+1, \omega_{t+1}$ subproblems, where stage $t+1$ is not balanced with stage $t+2$ (i.e., the stage $t+1$ cuts do not fully represent the stage $t+2$ expected costs), then the stage $t$ cuts are potentially weak cuts. They are not supports of the recourse function at the $x_{t}$ from wbich they were derived, but they have the potential to be supports of the recourse function at some other $x_{t}$. This situation is the case if in stage $t+1$, for a solution $\hat{x}_{t+1}^{\omega_{t+1}}, \hat{\theta}_{t+1}^{\omega_{t+1}}$

$$
\hat{\theta}_{t+1}^{\omega_{t+1}}<z_{t+2}\left(\hat{x}_{t+1}^{\omega_{t+1}}\right)
$$

The corresponding lower bound is then a weak lower bound.

If the cuts are obtained by Monte Carlo sampling rather than by solving the universe case, the optimal objective function value of the stage $t, \omega_{t}$ problem, $z_{t}^{\omega_{t}}$, represents an estimated lower bound for the stage $t, \omega_{t}$ expected costs (subject to $\hat{x}_{t-1}$ passed from the previous stage). The optimal objective function value of the stage 1 problem, $z_{1}$, represents an estimate for the lower bound of the expected costs of the multi-stage stochastic linear program.

In the following we derive a lower bound estimate for the multi-stage stochastic linear problem by analyzing the decomposed program, where at stages $t=1, \ldots, T-1$, $L_{t}$ cuts estimated by Monte Carlo (importance) sampling have been added. We start with an analysis of the relationship between stage $T-1$ and stage $T$. 
The Stage $T-1$ Master Problem:

$$
\begin{aligned}
& \begin{array}{c}
\tilde{z}_{T-1}^{\omega_{T-1}} \\
\mathrm{~s} / \mathrm{t}
\end{array}=\min c_{T-1} x_{T-1}^{\omega_{T-1}}+\theta_{T-1}^{\omega_{T-1}} \\
& \pi_{T-1}^{\omega_{T}}:=A_{T-1} x_{T-1}^{\omega_{T-1}}=b_{T-1}^{\omega_{T-1}}+B_{t-2}^{\omega_{T-1}} \hat{x}_{t} \\
& \rho_{T-1}^{1, \omega_{T-1}}: \quad-\bar{G}_{T-1}^{1} x_{T-1}^{\omega_{T-1}}+\theta_{T-1}^{\omega_{T-1}} \geq \bar{g}_{T-1}^{1}+\epsilon_{T-1}^{1}
\end{aligned}
$$

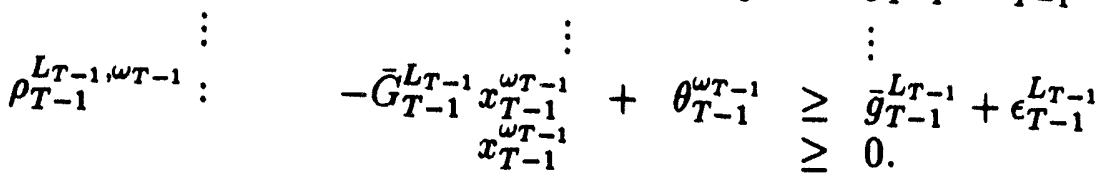

The Stage $T$ Subproblem:

$$
\begin{aligned}
\min z_{T}^{\omega_{T}}= & c_{T} x_{T}^{\omega_{T}} \\
\mathrm{~s} / \mathrm{t} & \\
\pi_{T}^{\omega_{T}}: & A_{T} x_{T}^{\omega_{T}}=b_{T}^{\omega_{T}}+B_{T-1}^{\omega_{T}} \hat{x}_{T-1} \\
& x_{T}^{\omega_{T}} \geq 0 .
\end{aligned}
$$

For a given scenario $\omega_{T-1}$ and a given solution $\hat{x}_{T-2}$ passed from the stage $T-1$ problem, the relation between stage $T-1$ and stage $T$ corresponds to that of a twostage stochastic linear program. We therefore can apply the theory which we have developed for solving two-stage stochastic linear programs directly.

In the stage $T-1$ problem, $L_{T-1}$ cuts have been added so far. These cuts have been obtained by passing solutions $\hat{x}_{T-1}^{l_{T-1}}, l_{T-1}=1, \ldots, L_{T-1}$, to stage $T$ and solving a sample set $S_{T}^{l_{T-1}}$ of stage $T$ subproblems according to the importance sampling scheme. By doing so we have obtained estimates of the stage $T$ expected costs $z_{T}\left(\hat{x}_{T-1}^{T-1}\right)$ and of the gradients $G_{T-1}^{l_{T-1}}$ and right-hand sides $g_{T-1}^{l_{T-1}}$ of the cuts. These estimates are denoted by $\bar{z}_{T}\left(\hat{x}_{T-1}^{l_{T-1}}\right), \bar{G}_{T-1}^{l_{T-1}}$ and $\bar{g}_{T-1}^{l_{T-1}}$. We also have obtained the error correction terms $\epsilon_{T-1}^{l_{T-1}}\left(x_{T-1}\right)$ based on the estimation error at $\hat{x}_{T-1}^{l_{T-1}}$, which for ease of exposition we assume to be constant with respect to $x_{t}$. In general their distributions vary with respect to $x_{T-1}$; see Section 7.2. However, assuming the error is constant with respect to $x_{T-1}$, each error term $\epsilon_{T-1}^{l_{T-1}}$ then arises from a normal distribution with mean 0 and variance $\operatorname{var}\left(\bar{z}_{T}\left(\hat{x}_{T-1}^{l_{T-1}}\right)\right)$. We denote

$$
\left(\sigma_{T-1}^{l_{T-1}}\right)^{2}:=\operatorname{var}\left(\bar{z}_{T}\left(\hat{x}_{T-1}^{l_{T-1}}\right)\right)
$$

Thus,

$$
\epsilon_{T-1}^{l_{T-1}}=N\left(0,\left(\sigma_{T-1}^{l_{T-1}}\right)^{2}\right)
$$

Based on a local error analysis of the stage $T-1$ problem (7.26) in scenario $\omega_{T-1}$ given $\hat{x}_{T-2}$, the optimal objective function value $\tilde{z}_{T-1}^{\omega_{T}-1}$ is a random parameter, normally distributed with mean $\bar{z}_{T-1}^{\omega_{T-1}}$ and variance $\operatorname{var}\left(\bar{z}_{T-1}^{\omega_{T-1}^{1}}\right)$,

$$
\tilde{z}_{T-1}^{\omega_{T-1}}=N\left(\bar{z}_{T-1}^{\omega_{T-1}}, \operatorname{var}\left(\bar{z}_{T-1}^{\omega_{T-1}}\right)\right)
$$


where

$$
\left(\sigma_{T-1}^{\omega_{T-1}}\right)^{2}:=\operatorname{var}\left(\bar{z}_{T-1}^{\omega_{T-1}}\right)=\sum_{l_{T-1}=1}^{L_{T-1}}\left(\rho_{T-1}^{\omega_{T-1}, l_{T-1}}\right)^{2}\left(\sigma_{T-1}^{l_{T-1}}\right)^{2} .
$$

The distribution of the stage $T-1$, scenario $\omega_{T-1}$ expected costs, $\tilde{z}_{T-1}^{\omega_{T-1}}$, is induced by the estimation errors in the cuts which have been obtained so far in stage $T-1$. The distribution is different for different scenarios $\omega_{T-1}$ due to the different dual variables $\rho_{T-1}^{\omega_{T-1}, l_{T-1}}$.

Next, we discuss the general case of the relation between stage $t-1$ and stage $t$, $t=2, \ldots, T-1$. In this case, the subproblems in stage $t$ contain cuts which have been computed by Monte Carlo importance sampling and therefore represent an estimate of the outer linearization of the future stage $t+1$ expected costs.

\section{The Stage $t$ Subproblem:}

$$
\begin{array}{rlrl}
\tilde{z}_{t}^{\omega_{t}}= & \min c_{t} x_{t}^{\omega_{t}}+\theta_{t}^{\omega_{t}} & \\
s / t & & =b_{t}^{\omega_{t}}+B_{t-1}^{\omega_{t}} \hat{x}_{t-1} \\
\pi_{t}^{\omega_{t}}: & & A_{t} x_{t}^{\omega_{t}} & \\
\rho_{t}^{1, \omega_{t}}: & -\bar{G}_{t}^{1} x_{t}^{\omega_{1}}+\theta_{t}^{\omega_{t}} & \geq \bar{g}_{t}^{1}+\epsilon_{t}^{1} \\
\rho_{t}^{L_{t}, \omega_{t}}: & \vdots & \vdots & \vdots \\
& -\bar{G}_{t}^{L_{t}} x_{t}^{\omega_{t}}+\theta_{t}^{\omega_{t}} & \geq \bar{g}_{t}^{L_{t}}+\epsilon_{t}^{L_{t}} \\
& x_{t}^{\omega_{t}} & & \geq 0 .
\end{array}
$$

In the analysis of the stage $T-1$ master problem we concluded that the objective value in stage $T-1$, scenario $\omega_{T-1}, \tilde{z}_{T-1}^{\omega_{T-1}}$, is normally distributed with mean $\bar{z}_{T-1}^{\omega_{T-1}}$ and variance $\left(\sigma_{T-1}^{\omega_{T-1}}\right)^{2}=\operatorname{var}\left(\bar{z}_{T-1}^{\omega_{T-1}}\right)$. In the following we show that for any stage $t$, $t=2, \ldots, T-2$, the expected costs $\tilde{z}_{t}^{\omega_{t}}$ are normally distributed with mean $\bar{z}_{t}^{\omega_{t}}$ and variance $\operatorname{var}\left(\bar{z}_{t}^{\omega_{t}}\right)$, which we will denote by $\left(\sigma_{t}^{\omega_{t}}\right)^{2}$ :

$$
\tilde{z}_{t}^{\omega_{t}}=N\left(\bar{z}_{t}^{\omega_{t}},\left(\sigma_{t}^{\omega_{t}}\right)^{2}\right)
$$

where

$$
\left(\sigma_{t}^{\omega_{t}}\right)^{2}:=\operatorname{var}\left(\bar{z}_{t}^{\omega_{t}}\right)=\sum_{l_{t}=1}^{L_{t}}\left(\rho_{t}^{\omega_{t}, l_{t}}\right)^{2}\left(\sigma_{t}^{l_{t}}\right)^{2}
$$

and

$$
\left(\sigma_{t}^{l_{t}}\right)^{2}:=\operatorname{var}\left(\bar{z}_{t+1}\left(\hat{x}_{t}^{l_{t}}\right)\right) \text {. }
$$

In order to do so, we discuss the relation between stage $t-1$ and stage $t$, where the stage $t, \omega_{t}$, problems are subproblems of the stage $t-1, \omega_{t-1}$ master problem. Given a solution $\hat{x}_{t-1}$ passed from the current stage $t-1, \omega_{t-1}$ master problem, the stage $t$ costs, $\tilde{\tilde{z}}_{t}$, are distributed both with respect to $\omega_{t}$ and with respect to the error distributions $\epsilon_{t}$ that appear at the right-hand sides of the cuts.

$$
\tilde{\tilde{z}}_{t}=\tilde{\tilde{z}}_{t}\left(\omega_{t}, \epsilon_{t}^{1}, \ldots, \epsilon_{t}^{L_{t}}\right) \text {. }
$$


The expected value of the stage $t$ costs with respect to the $\omega$ distribution, $\tilde{z}_{t}$, can be computed as

$$
\tilde{z}_{t}=E_{\omega_{t}}\left(\tilde{\bar{z}}_{t} \mid \omega_{t}\right)=E_{\omega_{t}}\left(\tilde{z}_{t}^{\omega_{t}}\right)
$$

using Monte Carlo importance sampling. Remember that $\tilde{z}_{t}^{\omega_{t}}=N\left(\bar{z}_{t}^{\omega_{t}}, \operatorname{var}\left(\bar{z}_{t}^{\omega_{t}}\right)\right)$. We compute the mean value $\tilde{z}_{t}^{\omega_{t}}$ by substituting the mean value of 0 of the error distributions $\epsilon_{t}^{l_{t}}$. That is, the current estimate of the mean is the current objective value.

We compute the variance $\operatorname{var}\left(\bar{z}_{t}\right)$ based on conditional expectations:

$$
\begin{aligned}
& \operatorname{var}\left(\tilde{\tilde{z}}_{t}\right)=E_{\omega_{t}}\left(\operatorname{var}\left(\tilde{\tilde{z}}_{t}\right) \mid \omega_{t}\right)+\operatorname{var}_{\omega_{t}}\left(E_{\epsilon_{t}} \tilde{\tilde{z}}_{t} \mid \omega_{t}\right) \\
& =E_{\omega_{t}} \operatorname{var}\left(\tilde{z}_{t}^{\omega_{t}}\right)+\operatorname{var}_{\omega_{t}}\left(\bar{z}_{t}^{\omega_{t}}\right) \\
& \operatorname{var}\left(\bar{z}_{t}\right)=\underbrace{}_{\left(\omega_{t}\right.} \operatorname{var}\left(\bar{z}_{t}^{\omega_{t}}\right)+\frac{1}{n} \operatorname{var}_{\omega_{t}} \bar{z}_{t}^{\omega_{t}} \\
& \text { future current. }
\end{aligned}
$$

The variance of the estimated mean of the stage $t$ costs can be seen as being composed of a sum of two terms. The first term concerns the influence of the error of the estimated cuts. It is an aggregate of the estimation error of all future periods, $t+2, \ldots, T$. We refer to it as the future term of the estimation error. For constant error distributions, $\operatorname{var}\left(\bar{z}_{t}^{\omega_{t}}\right)=\sum_{l_{t}=1}^{L_{t}}\left(\rho_{t}^{\omega_{t}, l_{t}}\right)^{2}\left(\sigma_{t}^{l_{t}}\right)^{2}$. When we consider the estimation error as dependent on $\hat{x}_{t}$, then $\operatorname{var}\left(\bar{z}_{t}^{\omega_{t}}\right)=\sum_{l_{t}=1}^{L_{t}}\left(\rho_{t}^{\omega_{t}, l_{t}}\right)^{2}\left(\sigma_{t}^{l_{t}}\left(\hat{x}_{t}\right)\right)^{2}$. The second term concerns the current estimation error due to using sampling in stage $t$. In the case of two-stage problems, only the second term appears because the subproblems do not contain estimated cuts representing future information. Given $\hat{x}_{t-1}$ passed from the stage $t-1$ master problem, we can define the variance of the estimate $\operatorname{var}\left(\bar{z}_{t}\right)$ to be

$$
\left(\sigma_{t-1}^{l_{t}-1}\right)^{2}:=\operatorname{var}\left(\bar{z}_{t}\left(\hat{x}_{t-1}^{l_{t-1}}\right)\right)
$$

Thus, the expected value of the stage $t$ costs is normally distributed with mean $\bar{z}_{t}\left(\hat{x}_{t-1}^{l_{t-1}}\right)$ and variance $\left(\sigma_{t-1}^{l_{t-1}}\right)^{2}$,

$$
\tilde{z}_{t}\left(\hat{x}_{t-1}^{l_{t-1}}\right):=N\left(\bar{z}_{t}\left(\hat{x}_{t-1}^{l_{t-1}}\right),\left(\sigma_{t-1}^{l_{t}-1}\right)^{2}\right) \text {. }
$$

The error correction terms of the stage $t-1$ cuts are represented as

$$
\epsilon_{t-1}^{l_{t-1}}=N\left(0,\left(\sigma_{t-1}^{l_{t-1}}\right)^{2}\right)
$$

where we assume that the error distribution is approximately constant with respect to $x_{t-1}$. The minimum costs in stage $t-1$, scenario $\omega_{t-1}, z_{t-1}^{\omega_{t-1}}$ are normally distributed with mean $\bar{z}_{t-1}^{\omega_{t-1}}$ and variance $\left(\sigma_{t-1}^{\omega_{t-1}}\right)^{2}$ :

$$
\tilde{z}_{t-1}^{\omega_{t-1}}=N\left(\bar{z}_{t-1}^{\omega_{t-1}},\left(\sigma_{t-1}^{\omega_{t-1}}\right)^{2}\right)
$$


Knowing the distribution of the error terms of the cuts in each stage $t, t=$ $2, \ldots, T-1$, we can compute an estimate of the mean of the stage $t$ costs and the corresponding estimated variance of the mean value of the costs.

At stage 1 we obtain an estimate $\bar{z}_{1}$ with corresponding variance $\operatorname{var}\left(\bar{z}_{1}\right)$, where we define

$$
\left(\sigma_{1}\right)^{2}:=\operatorname{var}\left(\bar{z}_{1}\right)
$$

and

$$
\left(\sigma_{1}\right)^{2}=\sum_{l_{1}=1}^{L_{1}}\left(\rho_{1}^{l_{1}}\right)^{2}\left(\sigma_{1}^{l_{1}}\right)^{2}
$$

The distribution of the optimal first stage costs,

$$
\tilde{z}_{1}=N\left(\bar{z}_{1},\left(\sigma_{1}\right)^{2}\right),
$$

represents a lower bound estimate for the expected costs of the multi-stage stochastic linear program.

\subsection{The Upper Bound Estimate}

To obtain an upper bound for the total expected costs of the multi-stage problem, we evaluate the expected costs of the current first-stage trial solution $\hat{x}_{1}$. This can be accomplished by sampling paths from stages $2, \ldots, T$. For a reference, see Pereira and Pinto (1989) [60]. To efficiently sample a small number of paths so as to obtain an accurate estimate of the expected costs associated with $\hat{x}_{1}$, we also use importance sampling. We define a path $\hat{s}^{\omega}=\left(\hat{x}_{1}, \hat{x}_{2}, \ldots, \hat{x}_{T}\right)^{\omega}, \omega \in \Omega$, where $\Omega=\left\{\Omega_{2} \times \Omega_{3} \times\right.$ $\left.\cdots \times \Omega_{T}\right\}$, as a sequence of optimal solutions $\hat{x}_{t}^{\omega_{t}}$ of stage $t$, scenario $\omega_{t}$ problems, $t=2, \ldots, T$, with $\hat{x}_{1}$ being the first-stage trial solution. A path is computed by following the "wait-and-see" paradigm. We pass $\hat{x}_{1}$ to the second stage and solve the second-stage problem for scenario $\omega_{2}$ to obtain the optimal solution $\hat{x}_{2}^{\omega_{2}}$. Next we pass the obtained second-stage solution $\hat{x}_{2}^{\omega_{2}}$ to the third-stage and solve the thirdstage problem for scenario $\omega_{3}$ to obtain $\hat{x}_{3}^{\omega_{3}}$. We continue in this way until we obtain $\hat{x}_{T}^{\omega_{T}}$ in stage $T$. Note that when solving the stage $t$ problem, no future outcomes $\omega_{t+1}, \ldots, \omega_{T}$ are used. All future information at each stage is solely represented by means of the cuts added in stage $t$ so far. The costs of a path $\hat{s}^{\omega}, C\left(\hat{s}^{\omega}\right)$, is given by $C\left(\hat{s}^{\omega}\right)=\sum_{t=1}^{T} c_{t} \hat{x}_{t}^{\omega_{t}}$. The expected value of the costs of all paths $\hat{s}^{\omega}, E \hat{s}^{\omega}$, gives an upper bound for the costs of a trial solution $\hat{x}_{1}$. Figure 2 represents schematically five paths sampled through the four-stage problem of Figure 1.

We sample paths by applying the importance sampling scheme to the space of dimension $\sum_{t=2}^{T} h_{t}$ of all random parameters $V_{i_{t}, t}, i_{t}=1, \ldots, h_{t}, t=2, \ldots, T$. For sampling paths the importance density $q(V)$ is computed based on the additive marginal approximation function $\Gamma(V)$ analogous to the way it was defined in Section 6:

$$
\Gamma(V)=C(\tau)+\sum_{t=1}^{T} \sum_{i_{t}=1}^{h_{t}} C\left(\tau_{1,1}, \ldots, \tau_{i_{t}-1, t}, V_{i_{t}, t}, \tau_{i_{1}+1, t}, \ldots, \tau_{h_{T}, T}\right)-C(\tau)
$$


where $V=\left(V_{1,1}, \ldots, V_{h_{1,1}}, V_{1,2}, \ldots, V_{h_{T}, T}\right)$ and $\tau=\left(\tau_{1,1}, \ldots, \tau_{h_{1,1},}, \tau_{1,2}, \ldots, \tau_{h_{T}, T}\right)$. Using importance sampling for the upper bound estimate, we hope to obtain accurate estimates with a small sample size. Note that the advantage of sampling paths lies in the fact that we only linearly increase the number of sample points with the number of stages, whereas the decision tree grows exponentially with the number of stages.

\section{The Algorithm}

By solving a sample of subproblems $\omega_{t+1}$ according to the importance sampling scheme, we compute estimates of the expected future costs $z_{t+1}^{\omega_{t}}$ and of the gradients $G_{t}^{l_{t}}$ and right-hand sides $g_{t}^{l_{t}}$ of the cuts in each stage $t$ and scenario $\omega_{t}$. The optimal objective function value for each stage $t$, scenario $\omega_{t}$ subproblem gives an estimated lower-bound for the expected costs $z_{t}^{\omega_{t}}=c_{t} \hat{x}_{t}^{\omega_{t}}+\hat{\theta}_{t}^{\omega_{t}}$, subject to scenario $\omega_{t}$ and subject to $\hat{x}_{t-1}$, the (optimal) solution passed forward from the previous stage. The obtained ' swer-bound estimate is the tightest lower bound that can be generated, if in stage $t+1$ a sufficient number of cuts have been added to represent the expected future costs with respect to stage $t+2$ for all scenarios $\omega_{t+1} \in \Omega_{t+1}$; it is a weaker lower-bound estimate if there is not a sufficient number of cuts.

We are especially interested in the lower-bound estimate of the first-stage costs, which we obtain by solving the first-stage problem. If the first-stage problem is balanced with the second stage (that is, if the cuts added so far to the first stage problem fully represent the expected second-stage costs), and if the second-stage is balanced with the third stage for all scenarios $\omega_{2} \in \Omega_{2}$ and all values of $\hat{x}_{1}$, and so forth until stage $T-1$, then the solution of the first-stage problem is the optimum solution of the multi-stage stochastic linear program. In this case, the lower bound estimate of $z_{1}$ takes on the value of the total expected costs of the multi-stage problem.

To estimate an upper bound for the total expected costs of the multi-stage problem, we use the path-sampling scheme with importance sampling to evaluate the expected costs of the current first-stage trial decision $\hat{x}_{1}$. Sampling paths $\omega \in \Omega$ according to this importance sampling scheme, we obtain an equal number of sample points $\omega_{t} \in \Omega_{t}$ in stages $t=2, \ldots, T$. Figure 3 represents schematically these sample points for the example of the five paths of Figure 2. At these sample points we define the current stage $t$ scenario $\omega_{t}$ subproblems and generate cuts to be added at stages $t=1, \ldots, T-1$, by employing importance sampling as described above for cuts.

The overall procedure works as follows. Solving the stage 1 problem at iteration 1 , we obtain a trial solution $\hat{x}_{1}$ and a lower bound estimate for the expected costs $z_{1}$. Now we employ the path sampling procedure to obtain an upper bound estimate for the expected costs $z_{1}$. If the upper bound estimate and the lower bound estimate are within a given optimality tolerance, we call the first-stage solution the optimal solution of the multi-stage problem and quit. Otherwise, we generate cuts in stages $1, \ldots, T-1$. The path-samrling procedure used for the upper bound estimate has produced sample points $\omega_{t} \in \Omega_{t}$ in stages $t=2, \ldots, T$, with corresponding ancestor 
solutions $\hat{x}_{1}$ and $\hat{x}_{t}^{\omega_{t}}$ in stages $t=2, \ldots, T-1$, to be passed to the current stage $t$ scenario $\omega_{\text {t }}$ problem. Starting at stage $T-1$ and moving backwards to stage 1 , we take each sample problem $\omega_{t}$ in stage $t$ and finally the stage 1 problem as the current master problem and compute cuts by again sampling $\omega_{t+1} \in \Omega_{t+1}$ descendant subproblems until each scenario problem $\omega_{t}$ in stage $t$ is balanced with stage $t+1$ with respect to ancestor solutions $\hat{x}_{t-1}$ which have been passed from stage $t-1$. Arriving at stage 1 , we obtain a new solution $\hat{x}_{1}$ and a new lower bound estimate. We continue as defined above by sampling new paths for the upper bound estimate. Finally, after a finite number of iterations, upper and lower bound estimates will be sufficiently close. Upper and lower bound estimates can be seen as sums of i.i.d. random terms, which for sample sizes of $\mathbf{3 0}$ or more can be assumed normally distributed with known variances (derived from the sampling process). A 95\% confidence interval for the obtained solution is computed.

\section{Computational Experience}

Computational results from using Benders decomposition and importance sampling for two-stage problems and a special class of multi-stage problems are discussed in Infanger (1992) [44] and Dantzig and Infanger (1991) [18], where we report on the solution of test problems with up to 52 stochastic parameters and a number of universe scenarios exceeding $10^{24}$. Using importance sampling and small sample sizes (between 100 and 600 ), very accurate results were obtained. Additional tests on these examples showed that the variance reduction factor obtained by using importance sampling versus crude (naive) Monte Carlo sampling was up to about $10^{-6}$.

Inspired by these results, we implemented an earlier version of the methodology described above for the multi-stage case : which did not consider dependency between stages. Instead of the path-sampling procedure for obtaining upper bound estimates, we implemented a procedure of sampling points which required handling of exponentially growing decision trees. Therefore, even when we used very small sample sizes, the number of stages that was practical to solve was limited.

We did test up to 3-stage problems. FI3 is a 3-stage test problem derived from a 2-stage financial portfolio problem found in Mulvey and Vladimirou (1989) [56]. The problem is to select a portfolio which maximizes expected returns in future periods, taking into account the possibility of revising the portfolio in each period. There are transaction costs and bounds on the holdings and turnovers. Our test problem covers a planning horizon of 3 periods whereas the original Mulvey-Vladimirou test problem was a 2-stage problem that compressed all future periods into a single second stage. They solved the stochastic problem by restricting the number of scenarios.

We assumed the returns of the stocks in the future periods to be independent stochastic parameters with 3 outcomes each. With 13 assets with uncertain returns, the problem had 26 stochastic parameters instead of 39 , because after the last-stage

decision is made, the expected money-value of the portfolio can be evaluated. The 
number of universe scenarios was $2.5 \cdot 10^{12}$. (The deterministic equivalent formulation of the problem would have more thin $\mathrm{i}^{14}$ rows and a similar number of columns.) We obtained an estimated optimal solution of the 3-stage stochastic problem using a sample size of only 50 per stage. The optimal objective function value was estimated to be 1.10895 with an estimated $95 \%$ confidence interval of $0.004 \%$ on the left side and $0.001 \%$ on the right side of the obtained objective function value. Thus the optimal objective value lies within $1.10881 \leq z^{*} \leq 1.10895$ with $95 \%$ probability. Note how small the confidence interval is.

\section{Conclusion}

We have developed the theory for solving a general class of multi-stage stochastic linear programs with dependency within stages and between stages. We discussed the theory of sharing cuts between different subproblems. We use Monte Carlo importance sampling techniques to obtain accurate estimates of expected values of both future costs and coefficients and right-hand sides of cuts, and derive probabilistic lower and upper bounds. The method involves importance path sampling for the upper bound estimation. It will enable us to efficiently solve large-scale multi-stage problems with many stages and numerous stochastic parameters in each stage. The implementation is subject to future research, preliminary computational results have turned out to be promising.

\section{Acknowledgments}

Since the author came to Stanford in 1989 as a visiting scholar from Vienna University of Technology he has been closely collaborating with Professor George B. Dantzig. The author wishes to thank George B. Dantzig for this collaboration and his outstanding professional support. The author also wants to thank David Morton and John Stone for valuable discusions regarding previous versions of this paper. 
Figures

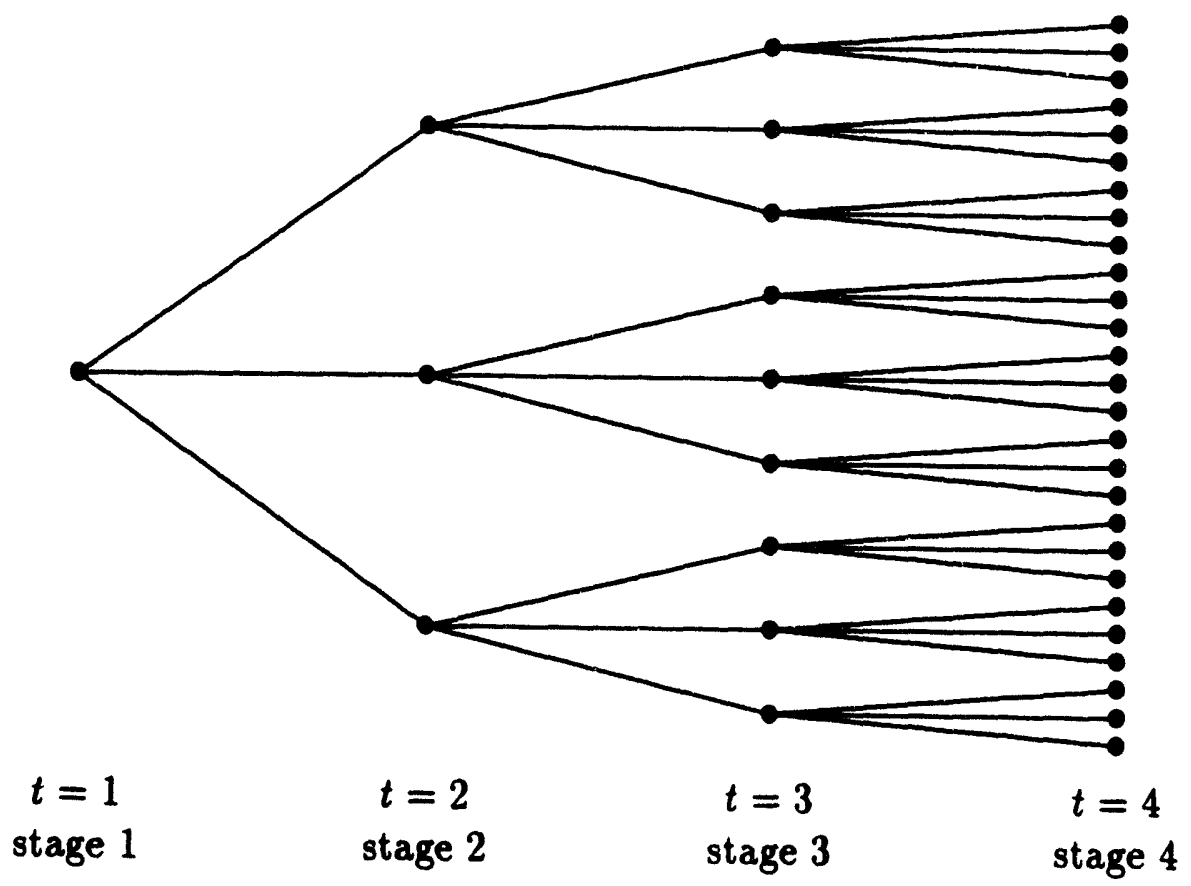

Figure 1: Decision tree of a general class of "wait and see" multi-stage problems 


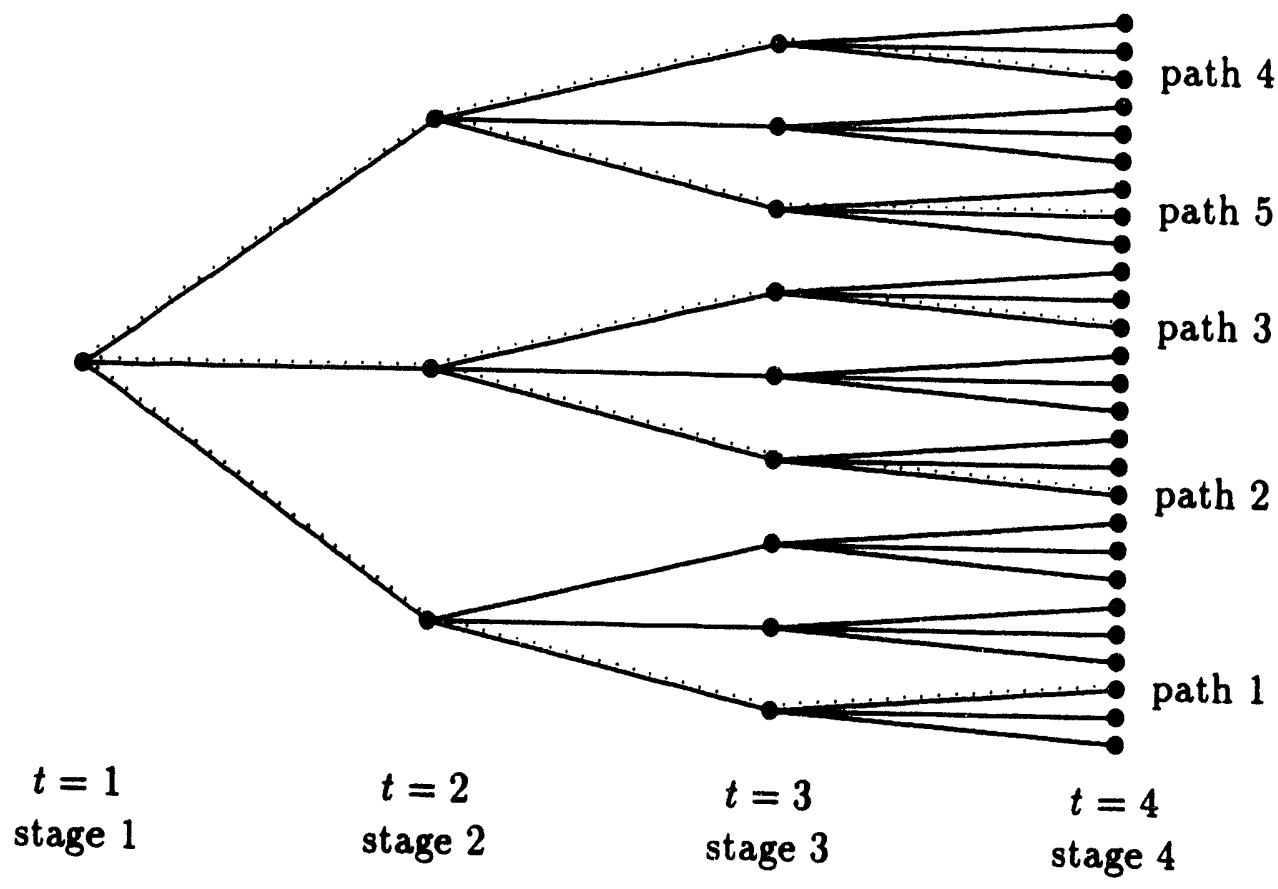

Figure 2: Path sampling for upper bounds

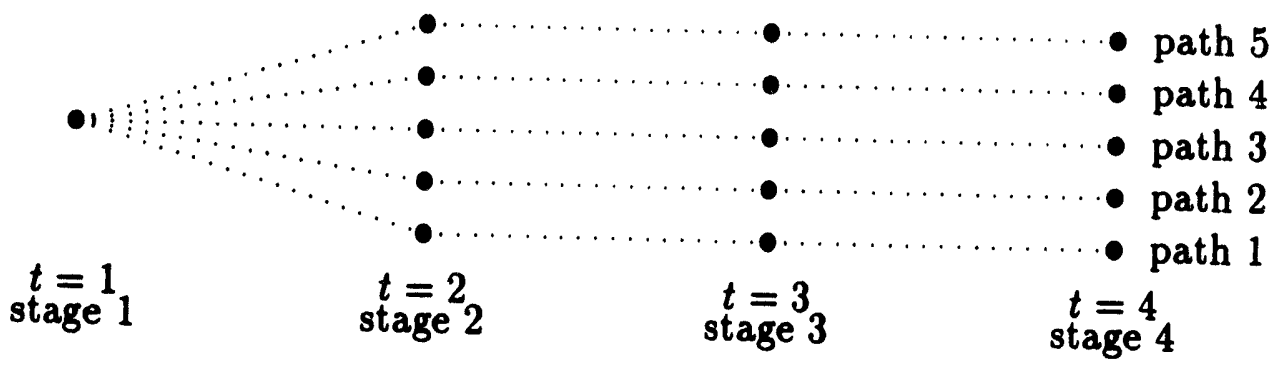

Figure 3: Sample points obtained from path sampling 


\section{References}

[1] Abrahamson, P.G. (1983): A Nested Decomposition Approach for Solving Staircase Linear Programs, Technical Report SOL 83-4, Department of Operations Research, Stanford University, Stanford, CA.

[2] Beale, E.M.L. (1955): On Minimizing a Convex Function Subject to Linear Inequalities, $J$. Roy. Stat. Soc. 176, 179-184.

[3] Benders, J.F. (1962): Partitioning Procedures for Solving Mixed-Variable Programming Problems, Numerische Mathematik 4, 298-252.

[4] Ben-Tal, A. and Hochman, E. (1972): More Bounds on the Expectation of a Random Variable, Journal of Applied Probability 9, 809-812.

[5] Birge, J.R. (1985): Decomposition and Partitioning Methods for Multi-Stage Stochastic Linear Programming, Operations Research 99, 989-1007.

[6] Birge, J.R. and Louveaux F.V. (1985): A Multicut Algorithm for Two-Stage Linear Programs, Technical Report, Department of IOE, University of Michigan, Ann Arbor, MI.

[7] Birge, J.R. and Wallace, S.W. (1988): A Separable Piecewise Linear Upper Bound for Stochastic Linear Programs, SIAM J. Control and Optimization 26, 9.

[8] Birge, J.R. and Wets, R.J. (1986): Designing Approximation Schemes for Stochastic Optimization Problems, in Particular For Stochastic Programs with Recourse, Math. Progr. Study 27, 54-102.

[9] Birge, J.R. and Wets, R.J. (1987): Computing Bounds for Stochastic Programming Problems by Means of a Generalized Moment Problem, Mathematics of Operations Research 12, 149-162

[10] Birge, J.R. and Wets, R.J. (1989): Sublinear Upper Bounds For Stochastic Programs with Recourse, Mathematical Programming 19, 191-119.

[11] Birge, J.R. and Wets, R.J. (1991) (eds): Stochastic Programming, Proceedings of the 5th International Conference on Stochastic Programming, Annals of Operations Research 90 and 91.

[12] Cipra, T. (1985): Moment Problem with Given Covariance Structure in Stochastic Programming, Ekonom.-Mat. Obzor 21, 66-77.

[13] Dantzig, G.B. (1955): Linear Programming under Uncertainty, Management Science 1, 197206.

[14] Dantzig, G.B. (1963): Linear Programming and Extensions, Princeton University Press, Princeton, NJ.

[15] Dantzig, C.P. and Glynn, P.W. (1990): Parallel Processors for Planning Under Uncertainty, Annals of Operations Research 22, 1-21.

[16] Dantzig, G.B., Ho, J.K. and Infanger, G. (1991): Solving Stochastic Linear Programs on a Hypercube Multicomputer, Technical Report SOL 91-10, Department of Operations Research, Stanford University, Stanford, CA.

[17] Dantzig, G.B., and Infanger, G. (1991): Multi-Stage Stochastic Linear Programs for Portfolio Optimization, Technical Report SOL 91-11, Department of Operations Research, Stanford University, Stanford, CA, to appear in Annals of Operations Research.

[18] Dantzig, G.B. and Infanger, G:(1991): Large-Scale Stochastic Linear Programs: Importance Sampling and Benders Decomposition, Technical Report SOL 91-4, Department of Operations Research, Stanford University, Stanford, CA. 
[19] Dantzig, G.B., and Infanger, G. (1993): Intelligent Control - Optimization under Uncertainty for the Control of Hydro Power Systems, Department of Operations Research, Stanford University, Stanford, CA, submitted to Annals of Operations Research.

[20] Dantzig, G.B. and Madansky, M. (1961): On the Solution of Two-Staged Linear Programs Under Uncertainty, Proc. Ath Berkeley Symp. on Mathematical Statistics and Probability I, ed. J. Neyman, 165-176.

[21] Dantzig, G.B. and Wolfe, P. (1960): The Decomposition Principle for Linear Programs, Operations Research 8, 110-111.

[22] Davis, P.J., and Rabinowitz, P. (1984): Methods of Numerical Integration, Academic Press, London.

[23] Dempster, M.A.H. (1980): Introduction to Stochastic Programming, in Dempster, M.A.H. (ed.): Stochastic Programming, Academic Press, 3-59.

[24] Dupačowá, J. (1978): Minimax Approach to Stochastic Linear Programming and the Moment Problem, Zeitschrift für Angewandte Mathematik und Mechanik, 58T, 466-167.

[25] Edmundson, H.P. (1956): Bounds on the Expectation of a Convex Function of a Random Variable, Paper 982, Rand Corporation, Santa Monica, CA.

[26] Ermoliev, Y. (1983): Stochastic Quasi-gradient Method3 and Their Applications to Systems Optimization, Stochastics 9, 1-96.

[27] Ermoliev, Y. (1988): Stochastic Quasi-gradient Methods, in Ermoliev, Y. and Wets, R.J-B. (eds.): Numerical Technigues for Stochastic Optimization, Springer Verlag, Berlin, Germany, 141-186.

[28] Ermoliev, Y. (1988): Stochastic Quasigradient Methods and their Implementation, in Ermoliev, Y. and Wets, R.J-B.. (eds.): Numerical Techniques for Stochastic Optimization, Springer Verlag, Berlin, Germany, 313-351.

[29] Ermoliev, Y. and Wets, R.J-B. (eds.) (1988): Numerical Techniques for Stochastic Optimization, Springer Verlag, Berlin, Germany.

[30] Frauendorfer, K. (1988): Solving SLP Recourse Problems with Arbitrary Multivariate Distributions-The Dependent Case, Mathematics of Operations Research 19, No. 9, 977994.

[31] Frauendorfer, K. (1992): Stochastic Two-Stage Programming, Lecture Notes in Economics and Mathematical Systems 392, Springer Verlag, Berlin, Germany.

[32] Frauendorfer, K. and Kall, P. (1988): Solving SLP Recourse Problems with Arbitrary Multivariate Distributions-The Independent Case, Problems of Control and Information Theory, Vol 17 (1), 177-205.

[33] Gaivoronski, A. (1988): Implementation of Stochastic Quasigradient Methods, in Ermoliev, Y. and Wets, R.J-B. (eds.): Numerical Techniques for Stochastic Optimization, Springer Verlag, Berlin, Germany.

[34] Gassmann, H. (1990): MSLiP: A Computer Code for the Multi-Stage Stochastic Linear Programming Problem, Mathematical Programming 47, 107-429.

[35] Gassmann, H. and Ziemba, W.T. (1986): A Tight Upper Bound for the Expectation of a Convex Function of a Multivariate Random Variable, Mathematical Programming Study 27, 99-58. 
[36] Geoffrion, A.M. (1974): Elements of Large-Scale Mathematical Programming, Management Science 16, No. 11.

[37] Glynn, P.W. and Iglehart, D.L. (1989): Importance Sampling for Stochastics Simulation, Management Science $95,1967-1992$.

[38] Huang, C.C., Ziemba, W.T. and Ben-Tal, A. (1977): Bounds on the Expectation of a Convex Function with a Random Variable with Applications to Stochastic Programming, Operations Research 25, 915-925.

[39] Hammersly, J.M. and Handscomb, D.C. (1964): Monte Carlo Methods, Mathuen, London.

[40] Hall, P. (1982): Rates of Convergence in the Central Limit Theorem, Research Notes in Mathematics 62.

[41] Higle, J.L. and Sen, S. (1989): Stochastic Decomposition: An Algorithm for Two-Stage Linear Programs with Recourse, Mathematics of Operations Research 16/9, 650-669.

[42] Higle, J.L. and Sen, S. (1989): Statistical Verification of Optimality Conditions for Stochastic Programs with Recourse, Annals of Operations Research 90, 215-210.

[43] Glassey, R. (1973): Nested Decomposition and Multi-Stage Linear Programs, Management Science 20, 282-292.

[44] Infanger, G. (1992): Monte Carlo (Importance) Sampling within a Benders Decomposition Algorithm for Stochastic Linear Programs, Annals of Operations Research 99, 69-95.

[45] Jensen, J.L. (1906): Sur les fonctions convexes et les inegalites entres les valeurs moyennes, Acta Mathematica 90, 175-199.

[46] Kall, P. (1974): Approximations to Stochastic Programs with Complete Fixed Recourse, Numer. Math. 22, 999-999.

[47] Kall, P. (1976): Stochastic Linear Programming, Springer Verlag, Berlin, Germany.

[48] Kall, P. and Stoyan, D. (1982): Solving Stochastic Programming problems with Recourse Including Error Bounds, Mathematische Operationsforschung und Statistik, Series Optimization, 19, 191-147.

[49] King, A. and Wets R.J-B. (1989): EPI-Consistency of Convex Stochastic Programs, submitted to Stochastics.

[50] Krishna, A.S. (1992): Importance Sampling Techniques for Stochastic Linear Programming, Dissertation in progress, Systems Optimization Laboratory, Department of Operations Research, Stanford University, Stanford, CA.

[51] Kusy, M.I. and Ziemba, W.T. (1986): A Bank Asset and Liability Management Model, Operations Research 91, 956-976.

[52] Louveaux, F.V. (1986): Multi-Stage Stochastic Programs with Block-Seperable Recourse, Mathematical Programming Study 28, 18-62.

[53] Lustig, I.J, Mulvey, J.M. and Carpenter, T.J. (1991): Formulating Two-Stage Stochastic Programs for Interior Point Methods, Operations Research 99, 757-770.

[54] Madansky, A. (1959): Bounds on the Expectation of a Convex Function of a Multivariate Random Variable, Annals of Mathematical Statistics 30, 7/9-7/6.

[55] Morton, D.P. (1992): Algorithmic Advances in Multistage Stochastic Programming, Dissertation in progress, Department of Operations Research, Stanford University, Stanford, CA. 
[56] Mulvey, J.M. and Vladimirou, H. (1989): Stochastic Network Optimization Models for Investment Planning, Annals of Operations Research 20 (1989) 187-217

[57] Mulvey, J.M. and Vladimirou, H. (1989): Applying the Progressive Hedging Algorithm to Stochastic Generalized Networks, Statistics and Operations Research Series Report, SOR-899, Princeton University, Princeton, New Jersey 08544.

[58] Mulvey, J.M. and Vladimirou, H. (1989): Solving Multi-Stage Stochastic Networks: An Application of Scenario Aggregation, Statistics and Operations Research Series Report, SOR-88-1, Department of Civil Engineering and Operations Research, Princeton University, Princeton, NJ.

[59] Murtagh, B.A. and Saunders, M.A. (1983): MINOS 5.0 User's Guide, SOL 83-20, Department of Operations Research, Stanford University, Stanford, CA.

[60] Pereira, M.V. and Pinto, L.M.V.G. (1989): Stochastic Optimization of a Multi-Reservoir Hydro-Electric System-A Decomposition Approach, Water Resources Research 21, 779-792.

[61] Pereira, M.V., Pinto, L.M.V.G., Oliveira, G.C. and Cunha, S.H.F. (1989): A Technique for Solving LP Problems with Stochastic Right-Hand Sides, CEPEL, Centro del Pesquisas de Energia Electria, Rio de Janeiro, Brazil.

[62] Pereira, M.V. and Pinto, L.M.V.G. (1989): Stochastic Dual Dynamic Programming, Technical Note, DEE-PUC/RJ, Catholic University of Rio de Janeiro, Rio de Janeiro, Brazil.

[63] Prékopa, A. (1988): Boole-Bonferroni Inequalities and Linear Programming, Operations Research 96, 145-162.

[64] Prékopa, A. (1989): Sharp Bounds on Probabilities Using Linear Programming, to appear in Operations Research.

[65] Prékopa, A. (1990): The Discrete Moment Problem and Linear Programming, Discrete Applied Mathematic 27, 295-254.

[66] Rockafellar, R.T. and Wets, R.J-B. (1989): Scenario and Policy Aggregation in Optimization Under Uncertainty, Mathematics of Operations Research 16, 119-117.

[67] Ruszczynski, A. (1986): A Regularized Decomposition Method for Minimizing a Sum of Polyhedral Functions, Mathematical Programming 95, 909-999.

[68] Van Slyke, R.M. and Wets, R.J-B. (1969): L-Shaped Linear Programs with Applications to Optimal Control and Stochastic Programming, SIAM J. of Applied Mathematics 17, 698-669.

[69] Wallace, S.W. (1987): A Piecewise Linear Upper Bound on the Network Recourse Problem, Mathematical Programming 98, 199-146.

[70] Wets, R.J-B. (1966): Programming under Uncertainty: The Complete Problem, Z. Wahrsch. verwo. Gebiete 4, \$16-\$s9.

[71] Wets, R.J-B. (1974): Stochastic Programs with Fixed Recourse: the Equivalent Deterministic Program, SIAM Review 16, 909-999.

[72] Wets, R.J-B. (1983): Solving Stochastic Programs with Simple Recourse, Stochastics 10, 219242.

[73] Wets, R.J-B. (1989): Stochastic Programming, in Nemhauser, G.L., Kan, A.H.G. and Todd, M.J. (eds.): Handbook on Operations Research and Management Science, North-Holland, Amsterdam, The Netherlands, 573-629.

[74] Wittrock, R.J. (1983): Advances in a Nested Decomposition Algorithm for Solving Staircase Linear Programs, Technical Report SOL 83-2, Department of Operations Research, Stanford University, Stanford, CA. 

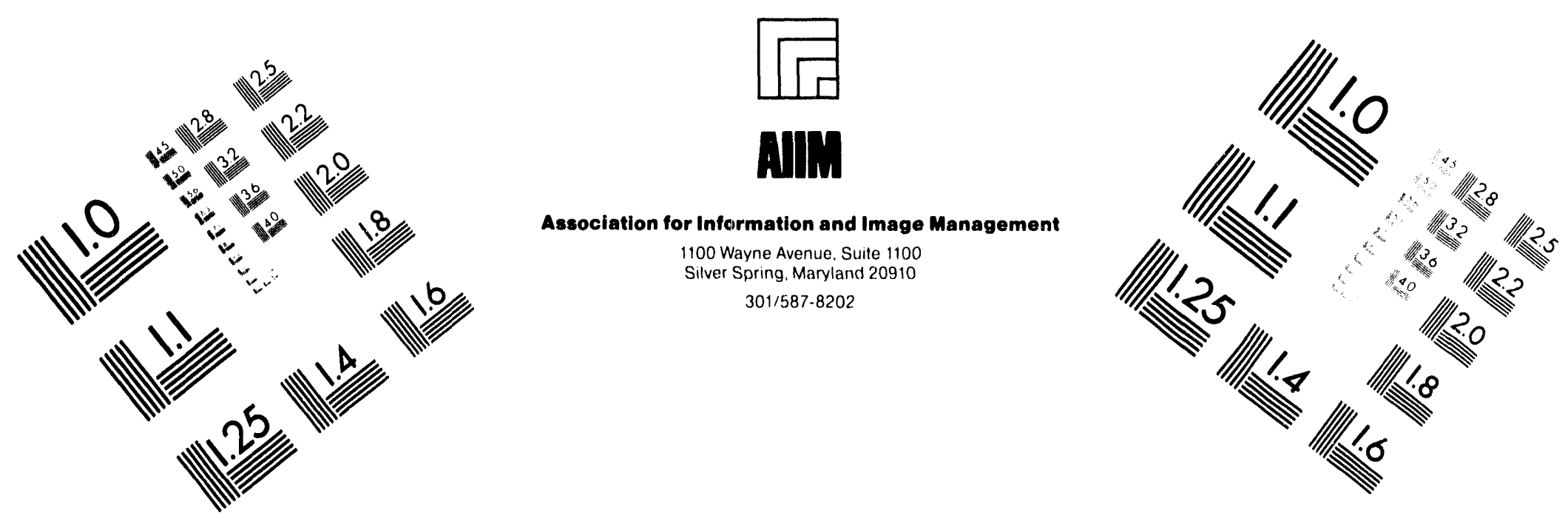

\section{Centimeter}

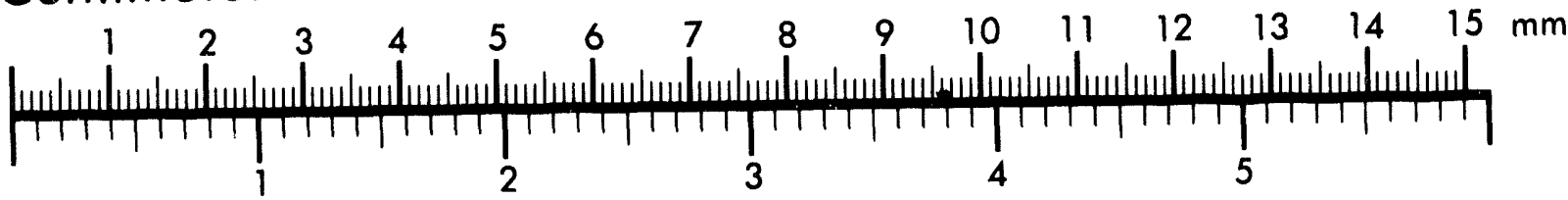
Inches
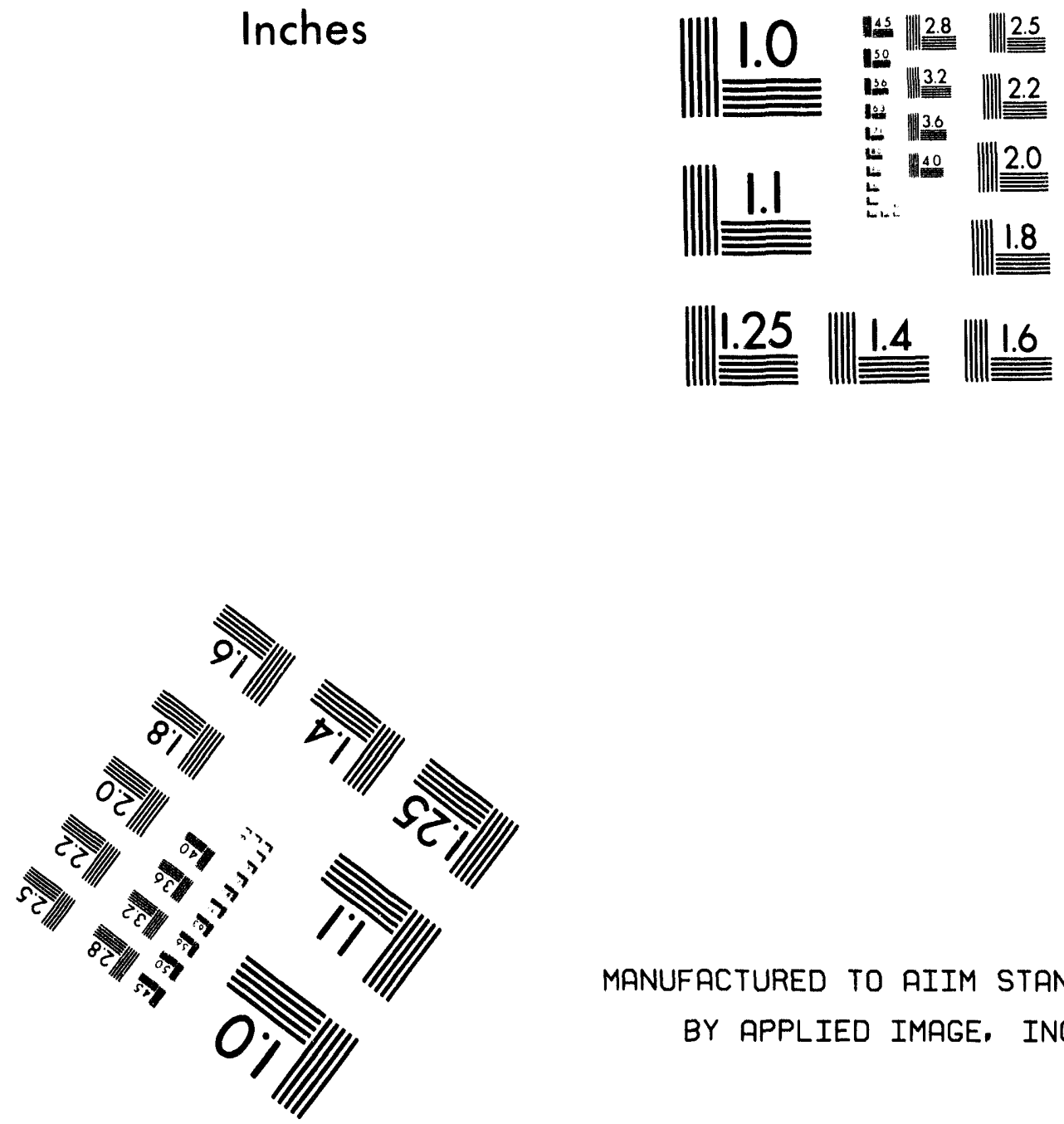

MANUFACTURED TO AIIM STANDARDS

BY APPLIED IMAGE, INC.

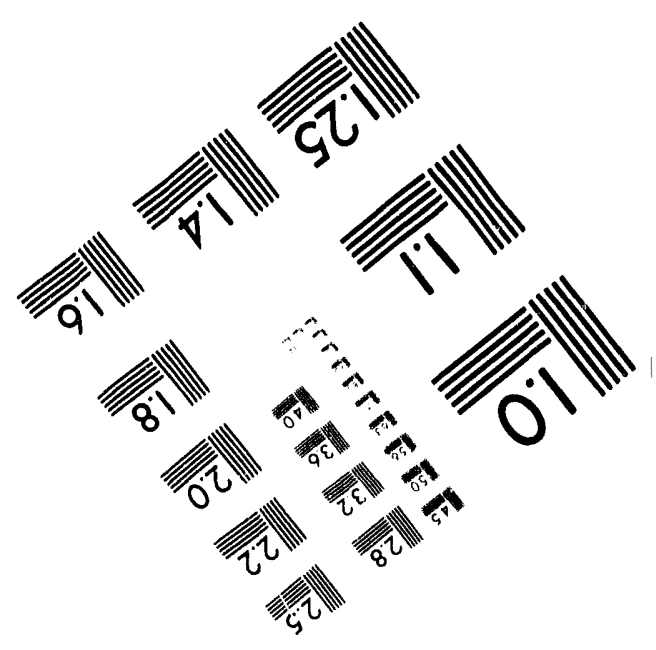


- 

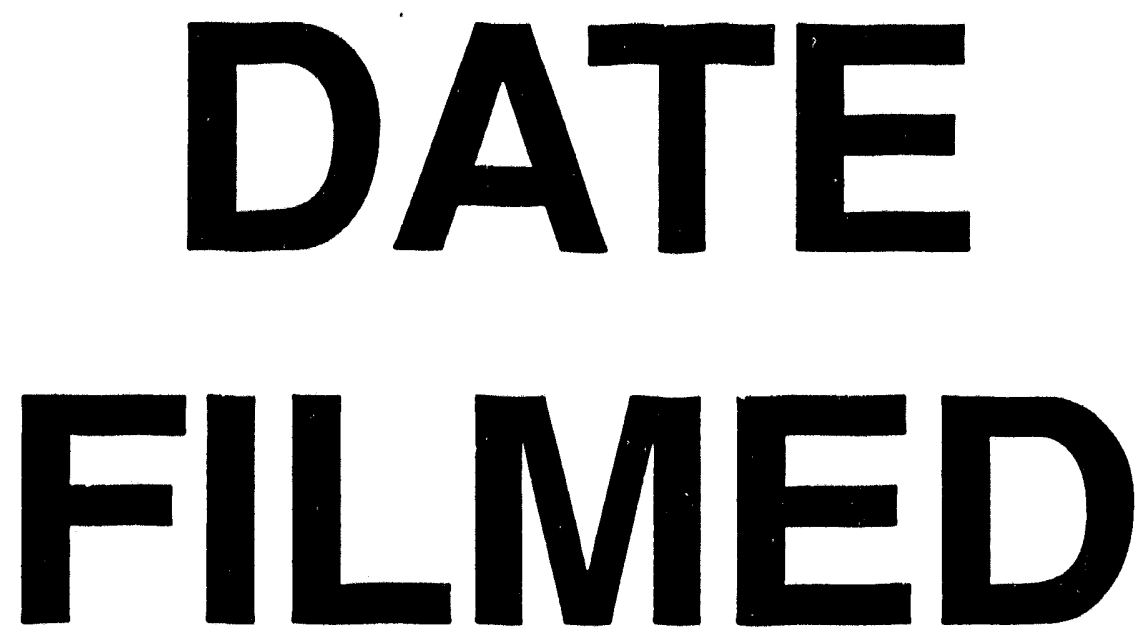

$12 / 28 / 94$
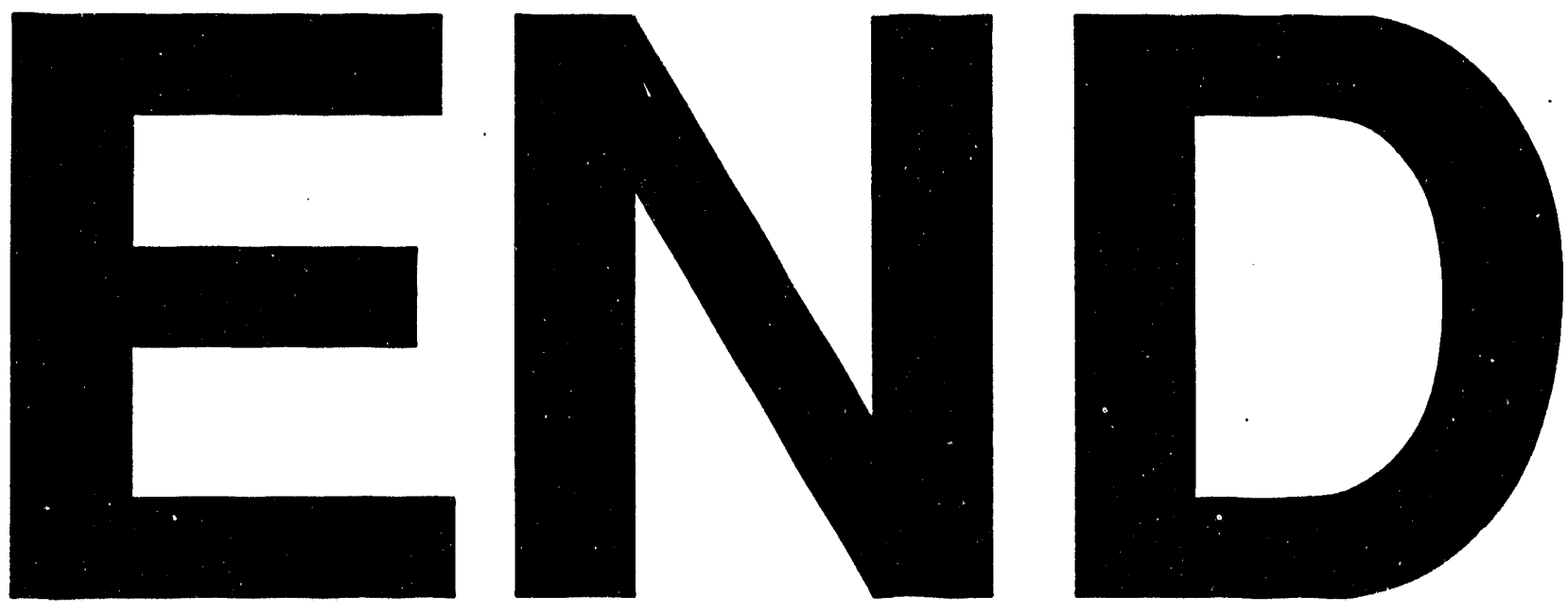